Article

\title{
Chemistry of Renieramycins. Part 19: Semi-Syntheses of 22-O-Amino Ester and Hydroquinone 5-O-Amino Ester Derivatives of Renieramycin $M$ and Their Cytotoxicity against Non-Small-Cell Lung Cancer Cell Lines
}

\author{
Supakarn Chamni ${ }^{1,2, * \mathbb{D}}$, Natchanun Sirimangkalakitti ${ }^{3}$, Pithi Chanvorachote ${ }^{4}$, \\ Khanit Suwanborirux ${ }^{2}$ and Naoki Saito ${ }^{3, *(1)}$ \\ 1 Natural Products and Nanoparticles Research Unit $\left(\mathrm{NP}^{2}\right)$, Chulalongkorn University, Bangkok 10330, Thailand \\ 2 Department of Pharmacognosy and Pharmaceutical Botany, Faculty of Pharmaceutical Sciences, Chulalongkorn \\ University, Pathumwan, Bangkok 10330, Thailand; Khanit.S@pharm.chula.ac.th \\ 3 Graduate School of Pharmaceutical Sciences, Meiji Pharmaceutical University, 2-522-1 Noshio, Kiyose, \\ Tokyo 204-8588, Japan; kikns@hotmail.com \\ 4 Department of Pharmacology and Physiology, Faculty of Pharmaceutical Sciences, Chulalongkorn University, \\ Pathumwan, Bangkok 10330, Thailand; pithi_chan@yahoo.com \\ * Correspondence: supakarn.c@pharm.chula.ac.th (S.C.); naoki@my-pharm.ac.jp (N.S.); Tel.: +66-218-8357 (S.C.); \\ $+81-424-95-8792$ (N.S.)
}

Received: 7 July 2020; Accepted: 8 August 2020; Published: 10 August 2020

\begin{abstract}
Two new series of synthetic renieramycins including 22-O-amino ester and hydroquinone 5-O-amino ester derivatives of renieramycin $\mathrm{M}$ were semi-synthesized and evaluated for their cytotoxicity against the metastatic non-small-cell lung cancer H292 and H460 cell lines. Interestingly, the series of 22-O-amino ester derivatives displayed a potent cytotoxic activity greater than the hydroquinone derivatives. The most cytotoxic derivative of the series was the 22-O-(N-Boc-L-glycine) ester of renieramycin $\mathrm{M}$ (5a: $\mathrm{IC}_{50} 3.56 \mathrm{nM}$ ), which showed 7-fold higher potency than renieramycin $\mathrm{M}$ ( $\mathrm{IC}_{50}$ $24.56 \mathrm{nM}$ ) and 61-fold more than jorunnamycin A ( $\mathrm{IC}_{50} 217.43 \mathrm{nM}$ ) against $\mathrm{H} 292$ cells. In addition, 5a exhibited a significantly higher cytotoxic activity than doxorubicin (ca. 100 times). The new semi-synthetic renieramycin derivatives will be further studied and developed as potential cytotoxic agents for non-small-cell lung cancer treatment.
\end{abstract}

Keywords: renieramycin M; bistetrahydroisoquinolinequinone; marine alkaloid; 22-O-amino ester derivatives of renieramycin $\mathrm{M}$; hydroquinone 5-O-amino ester derivatives of renieramycin $\mathrm{M}$; semi-synthesis; chemical modification; non-small-cell lung cancer; cytotoxicity

\section{Introduction}

Marine natural products have been documented as fascinating biologically active compounds. According to their structural complexity, marine molecules are capable of interacting with numerous biomolecular targets in the living cells to either inhibit or promote specific biological functions for therapeutic purposes [1]. Regarding the rich biodiversity of ocean creatures, several series of new natural products have been isolated from different marine organisms and reported as potential anti-cancer 
agents [2-4]. Several marine compounds and their synthetic derivatives are currently being investigated in different clinical trials $[5,6]$. To date, six marine-derived drugs have been approved by the United States Food and Drug Administration (U.S. FDA) including four anticancer drugs: cytarabine, eribulin mesylate, brentuximab vedotin, and trabectedin $[7,8]$.

Various known marine and microbial alkaloids belong to the tetrahydroisoquinoline family such as renieramycins, ecteinascidins, saframycins, safracins, naphthyridinomycins, and jorunnamycins. Interestingly, 1,2,3,4-tetrahydroisoquinoline analogs possess attractive anti-cancer activity against several cancer cell lines including leukemia, lung, and colon [9-12]. The 1,2,3,4-tetrahydroisoquinoline motif played an essential role as a minor groove DNA alkylator that covalently bonds specifically to the N2 position of guanine, which led to DNA bending toward the major groove and caused DNA damage in cancer cells [13]. These results have emphasized the importance of this naturally occurring 1,2,3,4-tetrahydroisoquinoline scaffold as a promising template for anti-cancer candidates.

Among the marine alkaloids, renieramycin M (1) is a marine bis-1,2,3,4-tetrahydroisoquinolinequinone alkaloid, which was isolated as a major alkaloid from the Thai blue sponge Xestospongia sp. found in the Si-Chang Island of Thailand (Figure 1) [14,15]. Interestingly, 1 and its semi-synthetic derivatives, replacing the angelate ester at various new ester side chains at C-22, exhibited a moderate to high potency of cytotoxicity in the nanomolar range against several human cancer cell lines such as colon, lung, and breast carcinomas [16]. In the past several years, the prominent structure-activity relationship studies of $\mathbf{1}$ and its semi-synthetic derivatives featuring various linear and aromatic ester side chains at C-22 and C-5 have been continuously explored and evaluated for their cytotoxic activity against non-small-cell lung cancer cell lines [17,18], which have been listed as one of the world's leading causes of death [19,20]. Based on the current findings, the chemically modified ester side chains at C-22 and C-5 displayed a key structure-cytotoxicity relationship. According to their cytotoxic potencies against non-small-cell lung cancer cell lines (H292 and H460), the nitrogen-containing aromatic esters such as the 4-pyridyl ester at C-22 and a small acyl ester such as the acetyl ester at C-5 are essential for the high potencies of $\mathbf{2}$ and 3, respectively. However, removing the $\mathrm{CN}$ group at $\mathrm{C}-21$ and the ester motif at $\mathrm{C}-22$ causes a drastically diminished cytotoxicity. The additional ester side chain at C-22 and C-5 presumably involves an increase in both hydrophobic interaction and the hydrogen bonding network during the DNA alkylation process to improve the cytotoxicity potency, similar to the mechanism of ecteinascidin 743 (trabectedin), which is an approved tetrahydroisoquinoline-containing antitumor drug. Additionally, the underlying anti-cancer mechanism of $\mathbf{1}$ and its semi-synthetic derivatives against non-small-cell lung cancer H23, H292, and H460 cell lines was reported to involve the apoptotic and anti-metastatic cell death pathways, which adds to the interest in this series of compounds as potential anti-non-small-cell lung cancer agents [21-26].
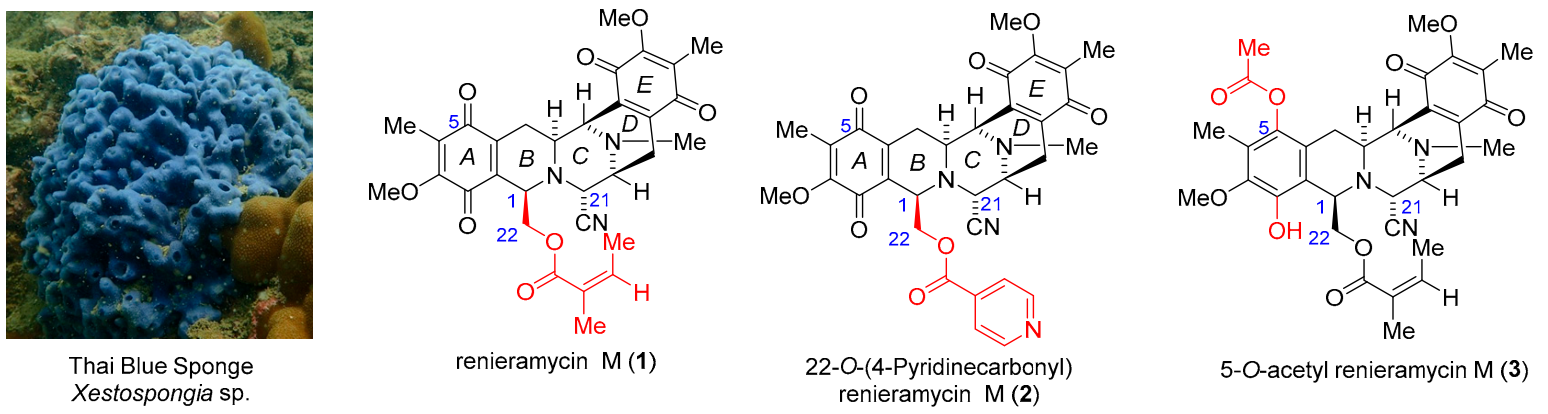

Figure 1. The Thai blue sponge Xestospongia sp., renieramycin $\mathrm{M}(\mathbf{1})$ and its semi-synthetic derivatives $(\mathbf{2}, \mathbf{3})$ with highly potent cytotoxicity. 
Based on the structural analysis of ecteinascidin 743 (4), the perpendicular ester bridge contains an aminoacyl moiety (red structure) on ring B of the tetrahydroisoquinoline core (Figure 2), which might be available for such interactions with other macromolecules, and two new series of the 22-O-amino ester and hydroquinone 5-O-amino ester derivatives of $\mathbf{1}$ (5 and 6) have been designed. This work is focused on the semi-synthesis of renieramycin $\mathrm{M}$ derivatives bearing an amino ester substituent at either the C-22 or C-5 positions and their cytotoxicity evaluations against the highly metastatic H292 and H460 non-small-cell lung cancer cell lines.

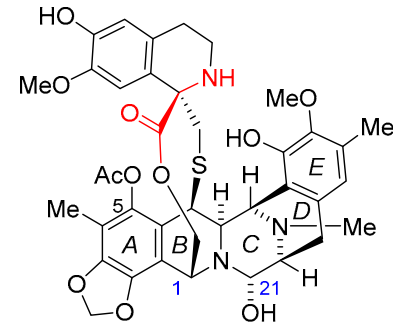

ecteinascidin $743(4)$

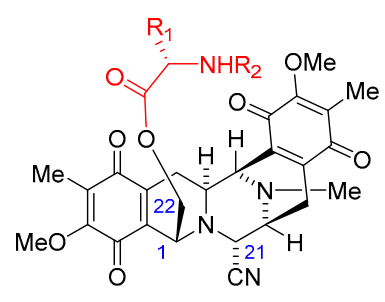

22-O-amino ester derivatives of renieramycin $\mathrm{M}(\mathbf{5})$

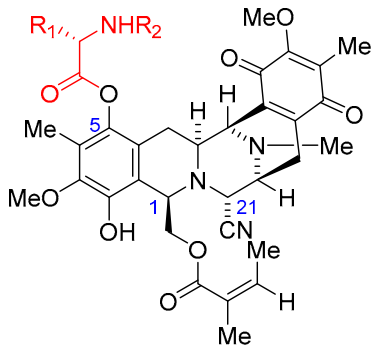

5-O-amino ester derivatives of renieramycin $\mathrm{M}(\mathbf{6})$

Figure 2. Ecteinascidin 743 as a template of the new series of 22-O-amino ester and hydroquinone 5-O-amino ester derivatives of $\mathbf{1}$.

\section{Results and Discussion}

\subsection{Extraction and Isolation of $\mathbf{1}$ from the Thai Blue Sponge Xestospongia sp.}

Compound 1 was isolated from the Thai blue sponge Xestospongia sp., collected from the vicinity of Si-Chang Island, the Gulf of Thailand. By using our optimized extraction process for the fresh blue sponge, regarding potassium cyanide pretreatment after neutralization at $\mathrm{pH} 7$ using phosphate buffer solution, 1 was obtained in a high yield [14]. In this work, a sample of the blue sponge Xestospongia sp. (ca. $18 \mathrm{~kg}$ wet weight equivalent to $3.5 \mathrm{~kg}$ dry weight) was collected. Extraction and purification afforded $\mathbf{1}(780 \mathrm{mg})$ with an isolation yield of $0.02 \% w / w$ relative to the dry sponge. Next, compound 1 was employed as the starting material for the semi-synthesis of the 22-O-amino ester and hydroquinone 5-O-amino ester derivatives.

\subsection{Syntheses of 22-O-amino Ester and Hydroquinone 5-O-amino Ester Derivatives of $\mathbf{1}$}

The preparation of 22-O-amino ester derivatives of $\mathbf{1}$ involved the transformation of $\mathbf{1}$ to jorunnamycin A (7) by the well-known three-step procedure including hydrogenation, hydride reduction, and air oxidation [16,27]. Next, 7 was reacted with the commercially available $N$-tert-butyloxycarbonyl (Boc)-protected amino acids including $N$-Boc-L-glycine, $N$-Boc-L-alanine, $N$-Boc-L-phenylalanine, N-Boc-L-valine, and N-Boc-L-proline under the Steglich esterification in the presence of 1-ethyl-3-(3-dimethylaminopropyl) carbodiimide (EDCI) as a coupling reagent to furnish the desired 22-O-amino ester derivatives of 1 in moderate to excellent yields (Scheme 1) [18].

The series of hydroquinone 5-O-amino ester derivatives was prepared by the hydrogenation of $\mathbf{1}$, yielding bishydroquinone renieramycin $\mathrm{M}(8)$, followed by esterification with the selected $\mathrm{N}$-Boc-L-amino acids and in situ air oxidation to afford the desired amino ester derivatives (Scheme 2). In more detail, the quinone motifs at rings $\mathrm{A}$ and $\mathrm{E}$ of $\mathbf{1}$ were chemoselectively reduced by the Pearlman's catalyst $\left(\mathrm{Pd}(\mathrm{OH})_{2} / \mathrm{C}\right)$ to yield bishydroquinone 8 with its angelate ester at C-22 remaining intact. The Steglich esterification was employed for the regiospecific esterification at the hydroxyl group on C-5, which was located at the least steric hindrance position. After esterification, the hydroquinone at ring $\mathrm{E}$ was restored by air oxidation to give back the quinone moiety, yielding the hydroquinone 5-O amino ester derivatives $(6)[15,18]$. Although 
the Steglich esterification with EDCI and N, N-4-dimethylaminopyridine (DMAP) was selected based on its mild and highly effective condition [28], the hydroquinone 5-O-amino ester derivatives were obtained at low to moderate yields due to the steric hindrances surrounding the hydroxyl group at C-5 of 8 and the $\mathrm{N}$-Boc-L-amino acid. Compound 1 was commonly recovered from each experiment (7-51\%). In addition, the yields of the hydroquinone 5-O-amino ester derivatives of 1 were increased when five equivalents of $\mathrm{N}$-Boc-L-amino acids were added in one portion. Furthermore, adjustment of the order of addition of 8 and N-Boc-L-amino acid was investigated. However, the results were not significantly different. Under these conditions, the major product $\mathbf{6}$ was formed through mono-esterification of hydroquinol at C-5.

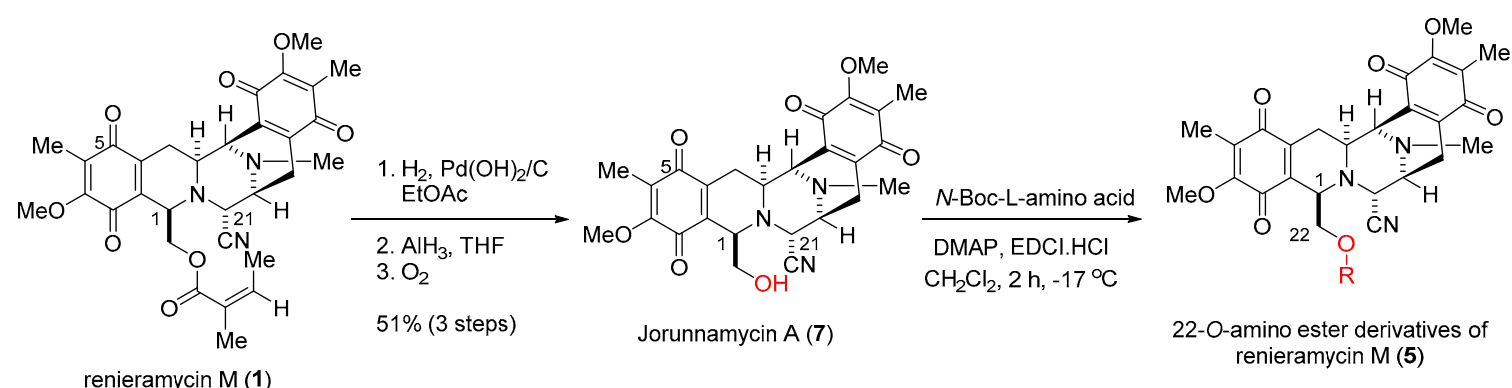
renieramycin $\mathrm{M}(\mathbf{1})$

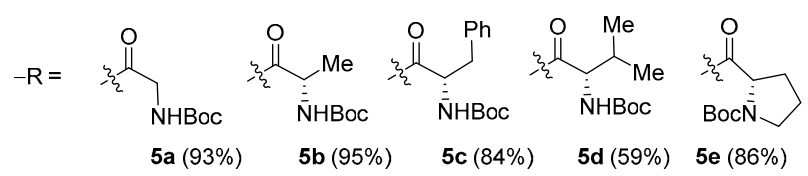

Scheme 1. Semi-synthesis of 22-O-amino ester derivatives of $\mathbf{1}(\mathbf{5 a}-\mathbf{5 e})$.

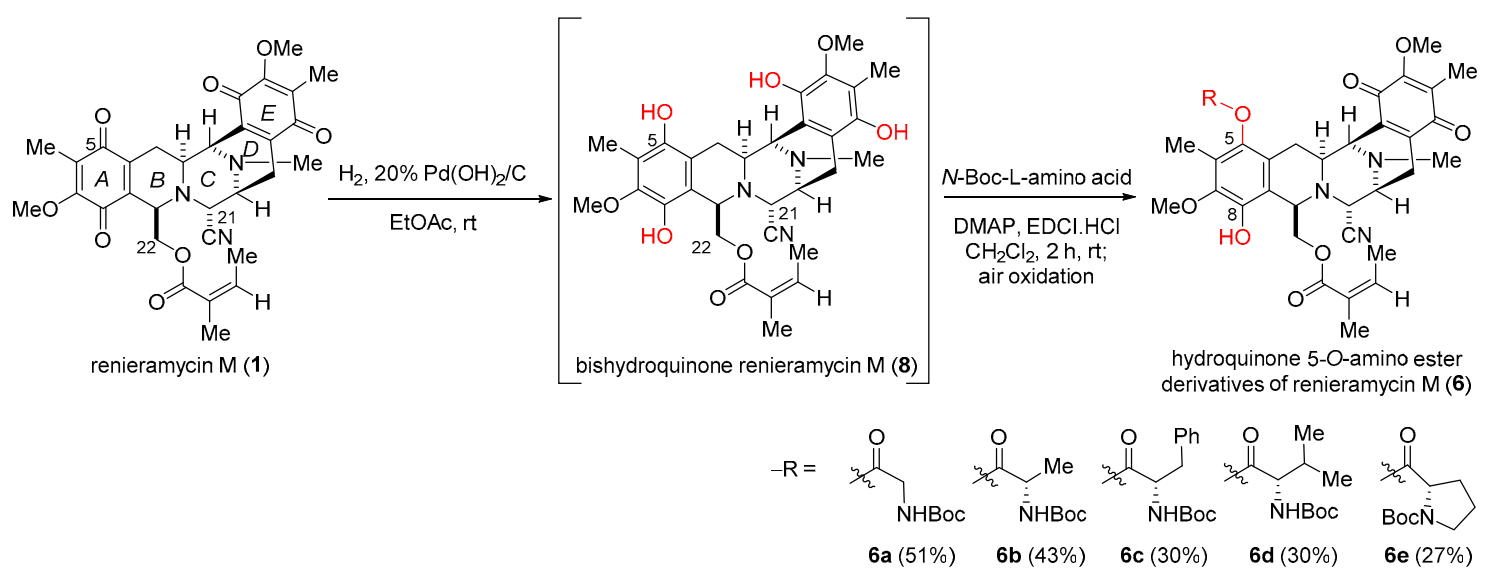

Scheme 2. Semi-synthesis of hydroquinone 5-O-amino ester derivatives of $\mathbf{1}$ (6a-6e).

\subsection{Cytotoxicity of the Semi-Synthetic Amino Ester Derivatives against Non-Small Cell Lung Cancer Cell Lines}

The cytotoxicity evaluations against aggressively metastatic H292 and H460 non-small-cell lung cancer cell lines were analyzed by an anti-proliferative assay using the tetrazolium dye, namely 3-(4,5-dimethylthiazol-2-yl)-2,5-diphenyltetrazolium bromide (MTT). As shown in Table 1, the 22-O-amino ester 5a-5e and hydroquinone 5-O-amino ester 6a-6e exhibited cytotoxicity in the nanomolar range from $\mathrm{IC}_{50} 3$ to $200 \mathrm{nM}$. Interestingly, the cytotoxicities of the newly synthesized derivatives in the amino ester series were significantly more potent than cisplatin and doxorubicin against the $\mathrm{H} 292$ cell line. In case of the H460 cell line, these amino ester derivatives exhibited a cytotoxic activity greater than cisplatin but showed a similar cytotoxicity to doxorubicin. Among the compounds in this series, 5a displayed the highest cytotoxic activity against the $\mathrm{H} 292$ cell line and was more potent than its parent compounds, 
which are 1 and 7 (7 times higher and 61 times higher), respectively (Table 1, entry 3). Additionally, 6a exhibited the strongest cytotoxic activity against the $\mathrm{H} 460$ cell line with a similar cytotoxicity to 1 and was 27 times more potent than 7 (Table 1, entry 8). Comparing 22-O-amino ester derivatives 5a-5e (Table 1, entries 3-7) and hydroquinone 5-O-amino ester derivatives 6a-6e (Table 1, entries 8-12), the series of 22-O-amino ester derivatives displayed significant cytotoxic activity against the $\mathrm{H} 292$ cell line, while the series of hydroquinone 5-O-amino ester derivatives were slightly more cytotoxic against the H460 cell line. Regarding the steric hindrance of the additional ester substituents, bulky groups such as phenylalanine and valine motifs, reduced the cytotoxic potencies, as exemplified by $\mathbf{5 c}$ and $\mathbf{6} \mathbf{d}$, respectively. It is worth pointing out that the steric hindrance played a larger part in the series of the hydroquinone 5-O-amino ester than the series of the 22-O-amino ester due to the inherited structural framework of the 1,2,3,4-tetrahydroisoquinolinequinone core.

Table 1. Cytotoxicity of 22-O-amino ester and hydroquinone 5-O-amino ester derivatives of 1.

\begin{tabular}{|c|c|c|c|c|}
\hline \multirow{2}{*}{ Entry } & \multirow{2}{*}{ Compound } & \multirow{2}{*}{ O-R } & H292 & H460 \\
\hline & & & $\mathrm{IC}_{50} \pm$ S.D. (nM) & $\mathrm{IC}_{50} \pm$ S.D. $(\mathrm{nM})$ \\
\hline 1 & 1 & Angeloyl & $24.56 \pm 1.12$ & $6.50 \pm 0.39$ \\
\hline 2 & 7 & $\mathrm{H}$ & $217.43 \pm 21.67$ & $164.30 \pm 11.07$ \\
\hline 3 & $5 a$ & N-Boc-L-Gly & $3.56 \pm 0.62$ & $9.94 \pm 0.82$ \\
\hline 4 & $5 b$ & N-Boc-L-Ala & $10.32 \pm 1.95$ & $19.75 \pm 2.38$ \\
\hline 5 & $5 c$ & N-Boc-L-Phe & $110.50 \pm 2.53$ & $51.43 \pm 5.67$ \\
\hline 6 & $5 d$ & $N$-Boc-L-Val & $32.39 \pm 3.33$ & $20.95 \pm 3.53$ \\
\hline 7 & $5 e$ & $N$-Boc-L-Pro & $66.55 \pm 9.47$ & $45.91 \pm 6.94$ \\
\hline 8 & $6 a$ & N-Boc-L-Gly & $10.24 \pm 0.94$ & $6.14 \pm 0.68$ \\
\hline 9 & $6 b$ & N-Boc-L-Ala & $24.03 \pm 2.61$ & $15.94 \pm 1.32$ \\
\hline 10 & $6 c$ & N-Boc-L-Phe & $57.35 \pm 5.36$ & $42.72 \pm 4.91$ \\
\hline 11 & $6 \mathrm{~d}$ & $N$-Boc-L-Val & $127.20 \pm 2.65$ & $200.53 \pm 29.27$ \\
\hline 12 & $6 e$ & $N$-Boc-L-Pro & $85.00 \pm 8.62$ & $43.28 \pm 0.68$ \\
\hline 13 & cisplatin & - & $12.13 \times 10^{3} \pm 1.12 \times 10^{3}$ & $8.15 \times 10^{3} \pm 0.64 \times 10^{3}$ \\
\hline 14 & doxorubicin & - & $350.70 \pm 32.94$ & $33.70 \pm 4.56$ \\
\hline
\end{tabular}

H292 and H460: human non-small cell lung cancer cell lines.

Considering both semi-synthesis and cytotoxicity results in this work, the 22-O-amino ester and hydroquinone 5-O-amino ester derivatives were structurally designed based on the addition of the aminoacyl moiety. This motif might play an interesting role in cytotoxicity through its interaction with other macromolecules during DNA alkylation. Moreover, the aminoacyl moiety with a small substituent such as the hydrogen and methyl group of glycine and alanine, respectively, displayed potent cytotoxicity. In contrast, a bulky substituent on the aminoacyl moiety exhibited lower cytotoxicity, as found in the isopropyl group of valine.

Initially, we planned to remove the Boc-protecting group by mild trifluoroacetic acid (TFA) deprotection. However, the preliminary Boc deprotection showed unsuccessful results regarding decomposition of the desired product. Thus, the Boc-protected derivatives were studied further against an antiproliferative MTT assay for cytotoxicity screening. Interestingly, the Boc group featuring a carbamate moiety and a bulky tertiary butyl group may be well suited as a model study to provide more information for future applications of such series of compounds as prodrugs $[29,30]$ or the cytotoxic payload with a linker for antibody conjugation [31], which requires a labile chemical moiety such as carbamate with bulky substitutes representing conjugated molecules. Based on the current investigation, $\mathbf{5 a}$ was obtained in an excellent yield (93\%) and exhibited a strong cytotoxicity greater than its parent compounds and the control 
drugs such as cisplatin and doxorubicin. Thus, $\mathbf{5 a}$ is a potential cytotoxic agent, which can be developed further for non-small cell lung cancer treatment and explored for its anti-cancer mechanism.

\section{Materials and Methods}

\subsection{General for Synthetic Procedure}

Reactions were carried out in oven-dried glassware and magnetically stirred under an argon atmosphere using a calcium chloride U-tube equipped with argon balloon, unless otherwise noted. Room temperature was $25^{\circ} \mathrm{C}$, unless otherwise stated. Commercial solvents and reagents were used as received, unless otherwise explained. Anhydrous solvents were dried over $4 \AA$ molecular sieves. Tetrahydrofuran (THF) was distilled immediately prior to use from the reaction of sodium metal and benzophenone. Brine refers to a saturated aqueous solution of sodium chloride. All reactions were monitored by thin-layer chromatography (TLC) performed using aluminum silica gel 60 F254 (Merck). Bands were identified by UV activity. Yields refer to chromatographically and spectroscopically pure compounds unless otherwise stated. Flash column chromatography was performed using $60 \AA$ silica gel (230-400 mesh) in a stationary phase. Optical rotations were measured on a Horiba-SEPA at $25^{\circ} \mathrm{C}$ in a 1-decimeter cell (length); concentration (c) is reported in $\mathrm{g} / \mathrm{mL}$, unless otherwise stated. Circular dichroism (CD) data were obtained on a JASCO J-720WI. IR spectra were measured on a Hitachi 260-10 spectrophotometer. The ${ }^{1} \mathrm{H}$ - and ${ }^{13} \mathrm{C}-\mathrm{NMR}$ spectra were recorded at $400 \mathrm{MHz}$ on a JEOL-JNM AL 400 FT-NMR spectrometer (supplementary files). The mass spectra were recorded on a JEOL-JMS 700 instrument with a direct inlet system operating at 70 eV. Accurate mass spectra were obtained with an Agilent 6540 UHD Q-TOF LC/MS spectrometer using fast atom bombardment $\left(\mathrm{FAB}^{+}\right)$ionization and a Zorbax Eclipse Plus C18 HPLC column $(3.0 \times 100 \mathrm{~mm})$. ${ }^{1} \mathrm{H}-\mathrm{NMR}$ chemical shifts are reported as $\delta$ values in ppm relative to tetramethylsilane (TMS, $0.00 \mathrm{ppm}$ ) or residual $\mathrm{CHCl}_{3}(7.27 \mathrm{ppm}) .{ }^{1} \mathrm{H}-\mathrm{NMR}$ coupling constants $(J)$ are reported in Hertz $(\mathrm{Hz})$. Unless otherwise indicated, deuterochloroform $\left(\mathrm{CDCl}_{3}\right)$ served as an internal standard $(77.0 \mathrm{ppm})$ for all ${ }^{13} \mathrm{C}$ spectra. The numbering of carbons in natural products and derivatives was adopted from initial isolation reports.

\subsection{Isolation of Renieramycin $M(\mathbf{1})$}

\subsubsection{Sample Collection}

The sponge Xestospongia sp. was collected by scuba diving in the vicinity of Si-Chang Island at a depth of 3-5 m with assistance from the Aquatic Recourses Research Institute, Chulalongkorn University and permission from the Department of Fisheries, Ministry of Agriculture and Cooperatives, Thailand (0510.2/8234, Date 28 October 2019). The fresh sponge was previously characterized based on its color and outer skeleton, including its light bluish-gray color, bulbous surface lobes, the numerous and moderate sizes of its oscules, and its easily crumbled texture [14]. The sample was kept frozen at $-20{ }^{\circ} \mathrm{C}$ until use.

\subsubsection{Extraction and Isolation}

The standard methods for isolation and purification of the blue sponge Xestospongia sp. involved potassium cyanide pre-treatment followed by methanolic extraction [14]. The collected marine organism, around $18 \mathrm{~kg}$, was homogenized and suspended in phosphate buffer solution ( $\mathrm{pH} 7)$. Then, a solution of $10 \%$ potassium cyanide in phosphate buffer solution $(\mathrm{pH} 7)$ was added dropwise to the suspension until it reached the concentration of potassium cyanide at $10 \mathrm{mM}$. During potassium cyanide pre-treatment step, the suspension was gradually checked and maintained at $\mathrm{pH} 7$ to avoid the formation of the toxic hydrogen cyanide gas. The mixture was continuously stirred for $5 \mathrm{~h}$. Thereafter, the mixture was macerated for $48 \mathrm{~h}$ with methanol. The extract was filtered, and the filtrate was concentrated under reduced pressure to obtain the aqueous methanolic solution. The maceration was repeated for 4 cycles. The combined 
aqueous methanolic solution was partitioned with hexane and ethyl acetate consecutively. In each partition, the relative volume of each organic solvent compared to the aqueous methanolic solution was 1:1 ratio. The obtained aqueous layers were discarded as the hazardous waste for further proper cyanide waste disposal [32]. Then, the volatile solvents were removed to give the crude residues. The ethyl acetate crude residue was subjected to silica gel chromatography using the gradient solvent mixture of hexane, ethyl acetate, and methanol. Finally, the purified products were combined and recrystallized in a 1:1 mixture of hexane and dichloromethane to obtain $\mathbf{1}(780 \mathrm{mg})$.

\subsection{Semi-Synthesis Procedures}

\subsubsection{Preparation of Jorunnamycin A (7)}

Compound 7 was semi-synthesized from 1 based on the reported protocol [27]. Compound 1 (100.0 $\mathrm{mg}, 0.1739 \mathrm{mmol})$ was weighed into the reaction vessel and dissolved in ethyl acetate (EtOAc) $(30 \mathrm{~mL})$. Then, to the resulting orange solution, $20 \% \mathrm{Pd}(\mathrm{OH})_{2}$ on carbon $(50.0 \mathrm{mg}, 50 \% w / w)$, was added. A hydrogen balloon was attached to the reaction flask. The heterogeneous reaction was stirred vigorously at room temperature $\left(25^{\circ} \mathrm{C}\right)$ under $1 \mathrm{~atm}$ for $5 \mathrm{~h}$. The reaction mixture was filtered through a pad of celite and washed with EtOAc $(20 \mathrm{~mL} \times 3)$ and $\mathrm{CHCl}_{3}(20 \mathrm{~mL} \times 3)$. The filtrates were combined and concentrated in vacuo to yield bishydroquinone renieramycin $\mathrm{M}$ as a colorless solid. The product was employed in the next step without further purification. A solution of bishydroquinone renieramycin $\mathrm{M}(83.1 \mathrm{mg}, 0.143 \mathrm{mmol})$ in dry THF $(10.0 \mathrm{~mL})$ was cooled at $-17^{\circ} \mathrm{C}$ and stirred in an argon atmosphere. A freshly prepared solution of $\mathrm{AlH}_{3}(0.5 \mathrm{M}, 2.3 \mathrm{~mL}, 1.147 \mathrm{mmol})$ was added dropwise over a $10 \mathrm{~min}$ period. The reaction mixture was stirred at $-17^{\circ} \mathrm{C}$ for $4 \mathrm{~h}$. The reaction was quenched by the addition of water $(2 \mathrm{~mL})$ and $\mathrm{CHCl}_{3}(20 \mathrm{~mL})$ at $-17^{\circ} \mathrm{C}$. The mixture was slowly warmed up to room temperature. After $20 \mathrm{~min}, \mathrm{Na}_{2} \mathrm{SO}_{4}(10 \mathrm{~g})$ was added and an oxygen balloon was attached to the reaction flask. The reaction mixture was stirred vigorously at room temperature overnight $(10 \mathrm{~h})$. After air oxidation, the reaction mixture was filtered and washed with $\mathrm{CHCl}_{3}(50 \mathrm{~mL} \times 3)$. The filtrates were combined and concentrated in vacuo until $20 \mathrm{~mL}$ of the crude mixture remained. The crude product was washed with $5 \% \mathrm{Na}_{2} \mathrm{CO}_{3}$ aqueous solution. The organic layer was separated by a separating funnel and the aqueous layer was extracted with $\mathrm{CHCl}_{3}(20 \mathrm{~mL} \times 3)$. The organic layers were combined, washed with brine $(50 \mathrm{~mL})$, dried over anhydrous $\mathrm{Na}_{2} \mathrm{SO}_{4}$, filtered, and concentrated in vacuo. Purification by silica gel flash chromatography and eluting with n-hexane:EtOAc (1:1 to $1: 9)$ gave $43.8 \mathrm{mg}$ ( $51 \%$ ) of 7 as an orange amorphous solid, along with $25.6 \mathrm{mg}$ (26\%) of recovered $\mathbf{1}$ and $5.8 \mathrm{mg}(7 \%)$ of decyanoyl jorunnamycin A. Jorunnamycin A (7); ${ }^{1} \mathrm{H}-\mathrm{NMR}\left(\mathrm{CDCl}_{3}, 400 \mathrm{MHz}\right) \delta 4.17$ $(1 \mathrm{H}, \mathrm{d}, J=2.1 \mathrm{~Hz}, 21-\mathrm{H}), 4.08(1 \mathrm{H}, \mathrm{d}, J=2.0 \mathrm{~Hz}, 11-\mathrm{H}), 4.03\left(3 \mathrm{H}, \mathrm{s}, 17-\mathrm{OCH}_{3}\right), 3.99\left(3 \mathrm{H}, \mathrm{s}, 7-\mathrm{OCH}_{3}\right), 3.89$ $(1 \mathrm{H}$, br d, $J=2.1 \mathrm{~Hz}, 1-\mathrm{H}), 3.71\left(1 \mathrm{H}, \mathrm{dd}, J=8.7,2.1 \mathrm{~Hz}, 22-\mathrm{H}_{\mathrm{a}}\right), 3.50\left(1 \mathrm{H}, \mathrm{br} \mathrm{d}, J=8.7 \mathrm{~Hz} 22-\mathrm{H}_{\mathrm{b}}\right), 3.42(1 \mathrm{H}$, br d, $J=5.0 \mathrm{~Hz}, 13-\mathrm{H}), 3.17(1 \mathrm{H}, \mathrm{dt}, J=8.5,2.0 \mathrm{~Hz}, 3-\mathrm{H}), 2.93\left(1 \mathrm{H}, \mathrm{dd}, J=13.0,2.0 \mathrm{~Hz}, 4-\mathrm{H}_{\alpha}\right), 2.83(1 \mathrm{H}$, $\left.\mathrm{dd}, J=16.0,5.0 \mathrm{~Hz}, 14-\mathrm{H}_{\alpha}\right), 2.30\left(3 \mathrm{H}, \mathrm{s}, \mathrm{NCH}_{3}\right), 2.27\left(1 \mathrm{H}, \mathrm{d}, J=16.0 \mathrm{~Hz}, 14-\mathrm{H}_{\beta}\right), 1.94\left(6 \mathrm{H}, \mathrm{s}, 6-\mathrm{CH}_{3}\right.$ and $\left.16-\mathrm{CH}_{3}\right), 1.41\left(1 \mathrm{H}, \mathrm{ddd}, J=13.0,8.5,1.8 \mathrm{~Hz}, 4-\mathrm{H}_{\beta}\right) ;{ }^{13} \mathrm{C}-\mathrm{NMR}\left(\mathrm{CDCl}_{3}, 100 \mathrm{MHz}\right) \delta 186.3(\mathrm{C}-15), 185.5(\mathrm{C}-5)$, 182.3 (C-18), 181.4 (C-8), 155.5 (C-7), 155.4 (C-17), 141.7 (C-20), 141.4 (C-10), 136.1 (C-9), 135.6 (C-19), 128.9 (C-6), $128.6(\mathrm{C}-16), 116.9(21-\mathrm{CN}), 64.3(\mathrm{C}-22), 61.1\left(17-\mathrm{OCH}_{3}\right), 61.1\left(7-\mathrm{OCH}_{3}\right), 59.1(\mathrm{C}-21), 58.0(\mathrm{C}-1), 54.5$ (C-13), $54.3(\mathrm{C}-3), 54.2(\mathrm{C}-11), 41.6\left(\mathrm{NCH}_{3}\right), 25.4(\mathrm{C}-4), 21.5(\mathrm{C}-14), 8.8\left(6-\mathrm{CH}_{3}\right), 8.7\left(16-\mathrm{CH}_{3}\right)$.

\subsubsection{General Procedure for Preparation of 22-O-amino Ester Derivatives of Renieramycin M (5a-5e)}

Compound 7 (10.0 mg, $0.02 \mathrm{mmol}, 1$ equivalent) was weighed in an oven dried-rounded bottom flask and dissolved in dry $\mathrm{CH}_{2} \mathrm{Cl}_{2}(2 \mathrm{~mL})$. The resulting orange solution was cooled at $-17{ }^{\circ} \mathrm{C}$ and 1-ethyl-3-(3-dimethylaminopropyl) carbodiimide (EDCI.HCl, $4.0 \mathrm{mg}, 0.02 \mathrm{mmol}, 1$ equivalent) and $\mathrm{N}, \mathrm{N}$-4-dimethylaminopyridine (DMAP, $3.0 \mathrm{mg}, 0.02 \mathrm{mmol}, 1$ equivalent) were added, followed by 
$\mathrm{N}$-Boc-L-amino acid ( $15-25 \mathrm{mg}, 0.10 \mathrm{mmol}, 5$ equivalent). The reaction mixture was stirred at $-17^{\circ} \mathrm{C}$ for $2 \mathrm{~h}$ in an argon atmosphere. The reaction mixture was quenched by the addition of water $(5 \mathrm{~mL})$. The organic layer was separated by a separating funnel and the aqueous layer was extracted with $\mathrm{CHCl}_{3}(10 \mathrm{~mL} \times 3)$. The organic layers were combined, washed with brine $(30 \mathrm{~mL})$, dried over anhydrous $\mathrm{Na}_{2} \mathrm{SO}_{4}$, filtered, and concentrated in vacuo. Purification by silica gel flash chromatography and eluting with n-hexane:EtOAc (4:1 to 2:1) gave 22-O-amino ester derivatives of $\mathbf{1}$.

22-O-(N-Boc-L-glycine) ester of renieramycin M (5a): $11.6 \mathrm{mg}(93 \%)$; yellow amorphous solid. $[\alpha]_{\mathrm{D}}^{25}$ +161.7 (c: $\left.0.0035, \mathrm{CHCl}_{3}\right)$; $\mathrm{CD} \Delta \varepsilon$ (c: $9 \mu \mathrm{M}$, methanol, $\left.20^{\circ} \mathrm{C}\right)+0.02(371),-0.3(314),-0.2(315),-1.0(300)$, -2.4 (283), -1.3 (269), -0.3 (257), -0.9 (242), -1.3 (227); IR (KBr) 3414, 2978, 2943, 2855, 1751, 1717, 1655, $1616,1508,1452,1369,1310,1281,1236,1161 \mathrm{~cm}^{-1} ;{ }^{1} \mathrm{H}-\mathrm{NMR}\left(\mathrm{CDCl}_{3}, 400 \mathrm{MHz}\right) \delta 4.84\left(1 \mathrm{H}, \mathrm{br} \mathrm{s}, 3^{\prime}-\mathrm{NH}\right)$, $4.47\left(1 \mathrm{H}, \mathrm{dd}, J=10.8,2.4 \mathrm{~Hz}, 22-\mathrm{H}_{\mathrm{a}}\right), 4.06(1 \mathrm{H}, \mathrm{br} \mathrm{d}, J=2.4 \mathrm{~Hz}, 21-\mathrm{H}), 4.03\left(3 \mathrm{H}, \mathrm{s}, 7-\mathrm{OCH}_{3}\right), 4.03(1 \mathrm{H}$, overlapped, 11-H), $4.01\left(3 \mathrm{H}, \mathrm{s}, 17-\mathrm{OCH}_{3}\right), 4.01(1 \mathrm{H}$, overlapped, 1- $\mathrm{H}), 3.95\left(1 \mathrm{H}\right.$, overlapped, 22- $\left.\mathrm{H}_{\mathrm{b}}\right), 3.78$ $\left(1 \mathrm{H}, \mathrm{dd}, J=18.6,6.0 \mathrm{~Hz}, 2^{\prime}-\mathrm{H}_{\mathrm{a}}\right), 3.49\left(1 \mathrm{H}, \mathrm{dd}, J=18.6,6.0 \mathrm{~Hz}, 2^{\prime}-\mathrm{H}_{\mathrm{b}}\right), 3.37(1 \mathrm{H}, \mathrm{br} \mathrm{d}, J=7.4 \mathrm{~Hz}, 13-\mathrm{H}), 3.10$ $(1 \mathrm{H}, \mathrm{dt}, J=12.0,2.6 \mathrm{~Hz}, 3-\mathrm{H}), 2.94\left(1 \mathrm{H}, \mathrm{dd}, J=17.3,2.6 \mathrm{~Hz}, 4-\mathrm{H}_{\alpha}\right), 2.73\left(1 \mathrm{H}, \mathrm{dd}, J=20.7,7.4 \mathrm{~Hz}, 14-\mathrm{H}_{\alpha}\right)$, $2.33\left(1 \mathrm{H}, \mathrm{d}, J=20.7 \mathrm{~Hz}, 14-\mathrm{H}_{\beta}\right), 2.30\left(3 \mathrm{H}, \mathrm{s}, \mathrm{NCH}_{3}\right), 1.96\left(3 \mathrm{H}, \mathrm{s}, 6-\mathrm{CH}_{3}\right), 1.95\left(3 \mathrm{H}, \mathrm{s}, 16-\mathrm{CH}_{3}\right), 1.42(9 \mathrm{H}, \mathrm{s}, 3 \times$ $\left.6^{\prime}-\mathrm{CH}_{3}\right), 1.35\left(1 \mathrm{H}\right.$, overlapped, $\left.4-\mathrm{H}_{\beta}\right) ;{ }^{13} \mathrm{C}-\mathrm{NMR}\left(\mathrm{CDCl}_{3}, 100 \mathrm{MHz}\right) \delta 186.6(\mathrm{C}-15), 185.2(\mathrm{C}-5), 182.4(\mathrm{C}-18)$, 181.1 (C-8), 169.8 (C-1'), 155.5 (C-4'), 155.4 (C-7), 155.3 (C-17), 142.1 (C-10), 142.0 (C-20), 135.1 (C-9), 135.0 (C-19), 128.9 (C-6), 128.3 (C-16), 116.9 (21-CN), $80.2\left(\mathrm{C}-5^{\prime}\right), 64.1$ (C-22), $61.1\left(17-\mathrm{OCH}_{3}\right), 61.1\left(7-\mathrm{OCH}_{3}\right), 59.0$ (C-21), 55.7 (C-1), $54.6(\mathrm{C}-3), 54.2(\mathrm{C}-13), 54.1(\mathrm{C}-11), 42.3\left(\mathrm{C}-2^{\prime}\right), 41.5\left(\mathrm{NCH}_{3}\right), 28.3\left(3 \times 6^{\prime}-\mathrm{CH}_{3}\right), 25.2(\mathrm{C}-4)$, $21.2(\mathrm{C}-14), 8.9\left(6-\mathrm{CH}_{3}\right), 8.6\left(16-\mathrm{CH}_{3}\right)$. FAB ${ }^{+} \mathrm{HRMS} m / z 651.6931\left([\mathrm{M}+\mathrm{H}]^{+}\right.$, calculated for $\mathrm{C}_{33} \mathrm{H}_{39} \mathrm{~N}_{4} \mathrm{O}_{10}$, 651.6929).

22-O-(N-Boc-L-alanine) ester of renieramycin M (5b): $12.3 \mathrm{mg}(95 \%)$; yellow amorphous solid. $[\alpha]_{\mathrm{D}}^{25}$ -75.8 (c: 0.0024, $\mathrm{CHCl}_{3}$ ); $\mathrm{CD} \Delta \varepsilon$ (c: $9 \mu \mathrm{M}$, methanol, $\left.20^{\circ} \mathrm{C}\right)-4.8$ (355), -3.5 (333), -2.1 (313), -2.8 (299), -9.3 (289), -16.1 (280), -5.0 (270), +8.9 (257), +5.4 (244), +0.2 (234); IR (KBr) 3393, 2978, 2940, 2855, 1765, 1717, 1653, 1616, 1506, 1456, 1369, 1306, 1234, 1155, $1058 \mathrm{~cm}^{-1} ;{ }^{1} \mathrm{H}-\mathrm{NMR}\left(\mathrm{CDCl}_{3}, 400 \mathrm{MHz}\right) \delta 4.69(1 \mathrm{H}$, $\left.\mathrm{d}, J=7.5 \mathrm{~Hz}, 3^{\prime}-\mathrm{NH}\right), 4.45\left(1 \mathrm{H}, \mathrm{br} \mathrm{d}, J=11.6 \mathrm{~Hz}, 22-\mathrm{H}_{\mathrm{a}}\right), 4.08\left(1 \mathrm{H}, \mathrm{br} \mathrm{d}, J=11.6 \mathrm{~Hz}, 22-\mathrm{H}_{\mathrm{b}}\right), 4.01(1 \mathrm{H}, \mathrm{d}$, $J=2.4 \mathrm{~Hz}, 21-\mathrm{H}), 3.96\left(3 \mathrm{H}, \mathrm{s}, 7-\mathrm{OCH}_{3}\right), 3.96\left(1 \mathrm{H}\right.$, overlapped, 11-H), $3.94\left(3 \mathrm{H}, \mathrm{s}, 17-\mathrm{OCH}_{3}\right), 3.92(1 \mathrm{H}$, overlapped, 1-H), $3.89\left(1 \mathrm{H}\right.$, overlapped, $\left.2^{\prime}-\mathrm{H}\right), 3.33(1 \mathrm{H}, \mathrm{br} \mathrm{d}, J=7.4 \mathrm{~Hz}, 13-\mathrm{H}), 3.02(1 \mathrm{H}, \mathrm{dt}, J=11.6,3.2 \mathrm{~Hz}$, 3-H), $2.82\left(1 \mathrm{H}, \mathrm{br} d, J=16.8, \mathrm{~Hz}, 4-\mathrm{H}_{\alpha}\right), 2.67\left(1 \mathrm{H}, \mathrm{dd}, J=20.9,7.4 \mathrm{~Hz}, 14-\mathrm{H}_{\alpha}\right), 2.30(1 \mathrm{H}, \mathrm{d}, J=20.9 \mathrm{~Hz}$, $\left.14-\mathrm{H}_{\beta}\right), 2.22\left(3 \mathrm{H}, \mathrm{s}, \mathrm{NCH}_{3}\right), 1.87\left(3 \mathrm{H}, \mathrm{s}, 6-\mathrm{CH}_{3}\right), 1.37\left(1 \mathrm{H}\right.$, overlapped, $\left.4-\mathrm{H}_{\beta}\right), 1.87\left(3 \mathrm{H}, \mathrm{s}, 16-\mathrm{CH}_{3}\right), 1.37(1 \mathrm{H}$, overlapped, $\left.4-\mathrm{H}_{\beta}\right), 1.31\left(9 \mathrm{H}, \mathrm{s}, 3 \times 6^{\prime}-\mathrm{CH}_{3}\right), 1.03\left(3 \mathrm{H}, \mathrm{d}, J=7.6 \mathrm{~Hz}, 7^{\prime}-\mathrm{CH}_{3}\right) ;{ }^{13} \mathrm{C}-\mathrm{NMR}\left(\mathrm{CDCl}_{3}, 100 \mathrm{MHz}\right) \delta$ 186.5 (C-15), 185.2 (C-5), 182.4 (C-18), 181.1 (C-8), 172.6 (C-1'), 155.6 (C-4'), 155.4 (C-7), 154.7 (C-17), 141.9 (C-10), 141.9 (C-20), 135.1 (C-9), 134.8 (C-19), 128.7 (C-6), 128.1 (C-16), 116.8 (21-CN), 79.9 (C-5'), 63.1 (C-22), $61.1\left(17-\mathrm{OCH}_{3}\right), 61.0\left(7-\mathrm{OCH}_{3}\right), 58.4(\mathrm{C}-21), 56.5(\mathrm{C}-1), 54.5(\mathrm{C}-3), 54.3(\mathrm{C}-13), 54.1(\mathrm{C}-11), 48.7\left(\mathrm{C}-2^{\prime}\right), 41.5$ $\left(\mathrm{NCH}_{3}\right), 28.2\left(3 \times 6^{\prime}-\mathrm{CH}_{3}\right), 25.2(\mathrm{C}-4), 21.0(\mathrm{C}-14), 18.3\left(7^{\prime}-\mathrm{CH}_{3}\right), 8.8\left(6-\mathrm{CH}_{3}\right), 8.5\left(16-\mathrm{CH}_{3}\right) . \mathrm{FAB}^{+} \mathrm{HRMS} \mathrm{m} / \mathrm{z}$ $665.2824\left([\mathrm{M}+\mathrm{H}]^{+}\right.$, calculated for $\left.\mathrm{C}_{34} \mathrm{H}_{41} \mathrm{~N}_{4} \mathrm{O}_{10}, 665.2823\right)$.

22-O-(N-Boc-L-phenylalanine) ester of renieramycin $\mathrm{M}(5 \mathrm{c})$ : $12.4 \mathrm{mg}(84 \%)$; yellow amorphous solid. $[\alpha]_{\mathrm{D}}^{25}+29.7\left(c: 0.0017, \mathrm{CHCl}_{3}\right) ; \mathrm{CD} \Delta \varepsilon\left(\mathrm{c}: 9 \mu \mathrm{M}\right.$, methanol, $\left.20^{\circ} \mathrm{C}\right)-6.7(356),-4.4(327),-4.0(300),-9.5$ (292), -23.1 (281), -3.5 (269), +11.4 (256), +5.4 (244), -2.8 (234); IR (KBr) 3387, 2963, 2928, 2855, 1719 (br), $1655,1612,1560,1508,1499,1458,1368,1306,1261,1234,1159,1107,1084,1049,1030,957,801,700 \mathrm{~cm}^{-1}$; ${ }^{1} \mathrm{H}-\mathrm{NMR}\left(\mathrm{CDCl}_{3}, 400 \mathrm{MHz}\right) \delta 7.29\left(3 \mathrm{H}, \mathrm{m} 2 \times 10^{\prime}-\mathrm{H} \& 11^{\prime}-\mathrm{H}\right), 7.05\left(2 \mathrm{H}, \mathrm{br} \mathrm{d}, J=6.3 \mathrm{~Hz}, 2 \times 9^{\prime}-\mathrm{H}\right), 4.71$ $\left(1 \mathrm{H}, \mathrm{d}, J=8.1 \mathrm{~Hz}, 3^{\prime}-\mathrm{H}\right), 4.30\left(2 \mathrm{H}, \mathrm{dd}, J=11.4,3.0 \mathrm{~Hz}, 22-\mathrm{H}_{2}\right), 4.24\left(1 \mathrm{H}, \mathrm{d}, J=7.8 \mathrm{~Hz}, 2^{\prime}-\mathrm{H}\right), 4.11(1 \mathrm{H}$, overlapped, 21-H), $4.08\left(1 \mathrm{H}\right.$, overlapped, 1-H), $4.00\left(6 \mathrm{H}, \mathrm{s}, 17\right.$ and $\left.7-\mathrm{OCH}_{3}\right), 3.87(1 \mathrm{H}, \mathrm{br}, 11-\mathrm{H}), 3.34(1 \mathrm{H}$, br d, $J=7.1 \mathrm{~Hz}, 13-\mathrm{H}), 3.07(1 \mathrm{H}, \mathrm{dt}, J=11.4,3.8 \mathrm{~Hz}, 3-\mathrm{H}), 2.91\left(1 \mathrm{H}, \mathrm{br} \mathrm{d}, J=16.8, \mathrm{~Hz}, 4-\mathrm{H}_{\alpha}\right), 2.84(2 \mathrm{H}, \mathrm{d}$, $\left.J=5.7 \mathrm{~Hz}, 7^{\prime}-\mathrm{H}\right), 2.45\left(1 \mathrm{H}, \mathrm{dd}, J=21.0,7.1 \mathrm{~Hz}, 14-\mathrm{H}_{\alpha}\right), 2.30\left(1 \mathrm{H}, \mathrm{d}, J=21.0 \mathrm{~Hz}, 14-\mathrm{H}_{\beta}\right), 2.28\left(3 \mathrm{H}, \mathrm{s}, \mathrm{NCH}_{3}\right)$, $1.92\left(3 \mathrm{H}, \mathrm{s}, 6-\mathrm{CH}_{3}\right), 1.86\left(3 \mathrm{H}, \mathrm{s}, 16-\mathrm{CH}_{3}\right), 1.38\left(1 \mathrm{H}\right.$, overlapped, $\left.4-\mathrm{H}_{\beta}\right), 1.32\left(9 \mathrm{H}, \mathrm{s}, 3 \times 66^{\prime}-\mathrm{CH}_{3}\right) ;{ }^{13} \mathrm{C}-\mathrm{NMR}$ $\left(\mathrm{CDCl}_{3}, 100 \mathrm{MHz}\right) \delta 186.4$ (C-15), 185.1 (C-5), 182.4 (C-18), 181.1 (C-8), 171.7 (C-1'), 155.5 (C-4'), 155.3 (C-7), 
154.7 (C-17), 142.2 (C-10), 141.9 (C-20), 135.7 (C-9), 134.9 (C-19), 129.3 (C-8'), $129.1\left(\mathrm{C}-9^{\prime}\right), 128.7$ (C-10'), 128.6 (C-6), $128.0(\mathrm{C}-16), 127.2\left(\mathrm{C}-11^{\prime}\right), 116.8(21-\mathrm{CN}), 80.0\left(\mathrm{C}-5^{\prime}\right), 64.3(\mathrm{C}-22), 62.0\left(\mathrm{C}-2^{\prime}\right), 61.1\left(17-\mathrm{OCH}_{3}\right)$, $61.0\left(7-\mathrm{OCH}_{3}\right), 58.6(\mathrm{C}-21), 55.9(\mathrm{C}-1), 54.6(\mathrm{C}-3), 54.3(\mathrm{C}-13), 54.2(\mathrm{C}-11), 41.4\left(\mathrm{NCH}_{3}\right), 38.3\left(\mathrm{C}-7^{\prime}\right), 28.1(3 \times$ $\left.6^{\prime}-\mathrm{CH}_{3}\right), 25.0(\mathrm{C}-4), 21.4(\mathrm{C}-14), 8.8\left(6-\mathrm{CH}_{3}\right), 8.6\left(16-\mathrm{CH}_{3}\right) . \mathrm{FAB}^{+} \mathrm{HRMS} m / z 741.3134\left([\mathrm{M}+\mathrm{H}]^{+}\right.$, calculated for $\left.\mathrm{C}_{40} \mathrm{H}_{45} \mathrm{~N}_{4} \mathrm{O}_{10}, 741.3136\right)$.

22-O-(N-Boc-L-valine) ester of renieramycin M (5d): $8.1 \mathrm{mg}(59 \%)$; yellow amorphous solid. $[\alpha]_{\mathrm{D}}^{25}$ +135.4 (c: 0.0023, $\left.\mathrm{CHCl}_{3}\right) ; \mathrm{CD} \Delta \varepsilon\left(\mathrm{c}: 6 \mu \mathrm{M}\right.$, methanol, $\left.20^{\circ} \mathrm{C}\right)-1.2(353),-0.8$ (326), -2.0 (301), -5.1 (289), -8.1 (281), -2.9 (270), +2.3 (257), -0.2 (240), -1.2 (224); IR (KBr) 3404, 2969, 2940, 1741, 1717, 1655, 1616, 1506, 1456, 1371, 1310, 1236, $1157 \mathrm{~cm}^{-1},{ }^{1} \mathrm{H}-\mathrm{NMR}\left(\mathrm{CDCl}_{3}, 400 \mathrm{MHz}\right) \delta 4.73\left(1 \mathrm{H}, \mathrm{br} \mathrm{d}, J=9.6 \mathrm{~Hz}, 3^{\prime}-\mathrm{H}\right)$, $4.29\left(2 \mathrm{H}, \mathrm{d}, J=2.7 \mathrm{~Hz}, 22-\mathrm{H}_{2}\right), 4.08(1 \mathrm{H}, \mathrm{d}, J=2.0 \mathrm{~Hz}, 21-\mathrm{H}), 4.07\left(1 \mathrm{H}\right.$, overlapped, $\left.2^{\prime}-\mathrm{H}\right), 4.06(3 \mathrm{H}, \mathrm{s}$, 7- $\left.\mathrm{OCH}_{3}\right), 4.04\left(1 \mathrm{H}\right.$, overlapped, 1-H), $4.03(1 \mathrm{H}$, overlapped, $11-\mathrm{H}), 4.00\left(3 \mathrm{H}, \mathrm{s}, 17-\mathrm{OCH}_{3}\right), 3.40(1 \mathrm{H}, \mathrm{dt}$, $J=7.8,2.0 \mathrm{~Hz}, 13-\mathrm{H}), 3.08(1 \mathrm{H}, \mathrm{dt}, J=11.4,2.4 \mathrm{~Hz}, 3-\mathrm{H}), 2.90(1 \mathrm{H}, \mathrm{dd}, J=16.8,2.4 \mathrm{~Hz}, 4-\mathrm{H} \alpha), 2.77(1 \mathrm{H}, \mathrm{dd}$, $\left.J=21.0,7.8 \mathrm{~Hz}, 14-\mathrm{H}_{\alpha}\right), 2.30\left(1 \mathrm{H}, \mathrm{d}, J=21.0 \mathrm{~Hz}, 14-\mathrm{H}_{\beta}\right), 2.28\left(3 \mathrm{H}, \mathrm{s}, \mathrm{NCH}_{3}\right), 1.95\left(3 \mathrm{H}, \mathrm{s}, 6-\mathrm{CH}_{3}\right), 1.93(3 \mathrm{H}$, $\left.\mathrm{s}, 16-\mathrm{CH}_{3}\right), 1.43\left(1 \mathrm{H}, \mathrm{d}, J=3.0 \mathrm{~Hz}, 4-\mathrm{H}_{\beta}\right), 1.36\left(9 \mathrm{H}, \mathrm{s}, 3 \times 6^{\prime}-\mathrm{CH}_{3}\right), 1.26\left(1 \mathrm{H}, \mathrm{m}, 7^{\prime}-\mathrm{H}\right), 0.80$ \& 0.73 (each $\left.3 \mathrm{H}, \mathrm{d}, J=6.9 \mathrm{~Hz}, 2 \times 8^{\prime}-\mathrm{CH}_{3}\right) ;{ }^{13} \mathrm{C}-\mathrm{NMR}\left(\mathrm{CDCl}_{3}, 100 \mathrm{MHz}\right) \delta 186.5(\mathrm{C}-15), 185.1(\mathrm{C}-5), 182.4(\mathrm{C}-18), 181.1$ (C-8), 171.8 (C-1'), 155.6 (C-4'), 155.5 (C-7), 155.2 (C-17), 141.9 (C-10), 141.8 (C-20), 135.1 (C-9), 134.8 (C-19), 128.5 (C-6), $127.8(\mathrm{C}-16), 116.7(21-\mathrm{CN}), 79.8\left(\mathrm{C}-5^{\prime}\right), 63.7(\mathrm{C}-22), 61.1\left(17-\mathrm{OCH}_{3}\right), 61.1\left(7-\mathrm{OCH}_{3}\right), 58.6\left(\mathrm{C}-2^{\prime}\right)$, 58.4 (C-21), 56.4 (C-1), 54.6 (C-3), $54.5(\mathrm{C}-13), 54.1(\mathrm{C}-11), 41.5\left(\mathrm{NCH}_{3}\right), 30.8\left(\mathrm{C}-7^{\prime}\right), 28.2\left(3 \times 6^{\prime}-\mathrm{CH}_{3}\right), 25.1$ (C-4), $21.2(\mathrm{C}-14), 18.9 \& 17.5\left(8^{\prime}-\mathrm{CH}_{3}\right), 8.8\left(6-\mathrm{CH}_{3}\right), 8.7\left(16-\mathrm{CH}_{3}\right) . \mathrm{FAB}^{+} \mathrm{HRMS} m / z 693.3143\left([\mathrm{M}+\mathrm{H}]^{+}\right.$, calculated for $\left.\mathrm{C}_{36} \mathrm{H}_{45} \mathrm{~N}_{4} \mathrm{O}_{10}, 693.3136\right)$.

22-O-(N-Boc-L-proline) ester of renieramycin $\mathrm{M}(5 \mathbf{e})$ : $11.6 \mathrm{mg}(86 \%)$; yellow amorphous solid. $[\alpha]_{\mathrm{D}}^{25}$ +161.1 (c: $\left.0.0044, \mathrm{CHCl}_{3}\right)$; $\mathrm{CD} \Delta \varepsilon$ (c: $14 \mu \mathrm{M}$, methanol, $\left.20^{\circ} \mathrm{C}\right)+1.6(417),-1.3(389),-3.8$ (358), $-2.6(328)$, -2.5 (300), -12.5 (282), -3.1 (270), +6.8 (258), +2.8 (243), -1.1 (222), +2.5 (212), +7.3 (207); IR (KBr) 2974, $2955,2940,1749,1695,1655,1616,1450,1400,1373,1312,1236,1188,1159,1084 \mathrm{~cm}^{-1}$; ${ }^{1} \mathrm{H}-\mathrm{NMR}\left(\mathrm{CDCl}_{3}\right.$, $400 \mathrm{MHz}) \delta 4.35\left(1 \mathrm{H}, \mathrm{dd}, J=11.6,2.0 \mathrm{~Hz}, 22-\mathrm{H}_{\mathrm{a}}\right), 4.29\left(1 \mathrm{H}, \mathrm{dd}, J=11.6,2.4 \mathrm{~Hz}, 22-\mathrm{H}_{\mathrm{b}}\right), 4.27\left(1 \mathrm{H}, \mathrm{m}, 2^{\prime}-\mathrm{H}\right)$, $4.10(1 \mathrm{H}, \mathrm{br}, 21-\mathrm{H}), 4.01\left(1 \mathrm{H}\right.$, overlapped, 11-H), $4.02\left(3 \mathrm{H}, \mathrm{s}, 7-\mathrm{OCH}_{3}\right), 3.99\left(3 \mathrm{H}, \mathrm{s}, 17-\mathrm{OCH}_{3}\right), 3.99(1 \mathrm{H}$, overlapped, 1-H), $3.40(1 \mathrm{H}, \mathrm{m}, 13-\mathrm{H}), 3.30\left(2 \mathrm{H}, \mathrm{m}, 9^{\prime}-\mathrm{H}_{2}\right), 3.09(1 \mathrm{H}$, br d, $J=11.6 \mathrm{~Hz}, 3-\mathrm{H}), 2.95(1 \mathrm{H}$, br $\left.\mathrm{d}, J=17.2 \mathrm{~Hz}, 4-\mathrm{H}_{\alpha}\right), 2.76\left(1 \mathrm{H}, \mathrm{dd}, J=20.7,7.6 \mathrm{~Hz}, 14-\mathrm{H}_{\alpha}\right), 2.33\left(1 \mathrm{H}, \mathrm{d}, J=20.7 \mathrm{~Hz}, 14-\mathrm{H}_{\beta}\right), 2.28(3 \mathrm{H}, \mathrm{s}$, $\left.\mathrm{NCH}_{3}\right), 1.93\left(3 \mathrm{H}, \mathrm{s}, 6-\mathrm{CH}_{3}\right), 1.91\left(3 \mathrm{H}, \mathrm{s}, 16-\mathrm{CH}_{3}\right), 1.75\left(2 \mathrm{H}, \mathrm{m}, 8^{\prime}-\mathrm{H}_{2}\right), 1.34\left(9 \mathrm{H}, \mathrm{s}, 3 \times 6^{\prime}-\mathrm{CH}_{3}\right), 1.43(1 \mathrm{H}$, overlapped, $\left.4-\mathrm{H}_{\alpha}\right), 1.26\left(2 \mathrm{H}, \mathrm{m}, 7^{\prime}-\mathrm{H}_{2}\right) ;{ }^{13} \mathrm{C}-\mathrm{NMR}\left(\mathrm{CDCl}_{3}, 100 \mathrm{MHz}\right) \delta 186.4(\mathrm{C}-15), 185.3(\mathrm{C}-5), 182.4$ (C-18), 181.1 (C-8), 172.5 (C-1'), 155.7 (C-4'), 155.5 (C-7), 154.0 (C-17), 142.0 (C-10), 141.8 (C-20), 135.3 (C-9), 134.7 (C-19), 128.4 (C-6), 127.8 (C-16), 117.0 (21-CN), 79.7 (C-5'), 65.2 (C-22), 63.7 (C-2'), 61.0 (17-OCH $\mathrm{OC}_{3}$ \& 7-OCH 3 ), 58.5 (C-21), 58.6 (C-4), 56.4 (C-1), 54.6 (C-3), 54.3 (C-11), $54.2(\mathrm{C}-13), 46.5\left(\mathrm{C}-9^{\prime}\right), 41.5\left(\mathrm{NCH}_{3}\right), 31.6$ $\left(\mathrm{C}-7^{\prime}\right), 29.8,\left(\mathrm{C}-8^{\prime}\right), 28.3\left(3 \times 6^{\prime}-\mathrm{CH}_{3}\right), 25.2(\mathrm{C}-4), 21.2(\mathrm{C}-14), 8.8\left(6-\mathrm{CH}_{3}\right), 8.6\left(16-\mathrm{CH}_{3}\right) . \mathrm{FAB}^{+} \mathrm{HRMS} \mathrm{m} / \mathrm{z}$ $691.2970\left([\mathrm{M}+\mathrm{H}]^{+}\right.$, calculated for $\left.\mathrm{C}_{36} \mathrm{H}_{43} \mathrm{~N}_{4} \mathrm{O}_{10}, 691.2980\right)$.

3.3.3. General Procedure for Preparation of Hydroquinone 5-O-amino Ester Derivatives of Renieramycin $\mathrm{M}(\mathbf{6 a}-6 \mathrm{e})$

Compound 1 (37.0 mg, $0.065 \mathrm{mmol})$ was dissolved in EtOAc $(20 \mathrm{~mL})$ and $20 \% \mathrm{Pd}(\mathrm{OH})_{2}$ on carbon $(19.0 \mathrm{mg}, 50 \% \mathrm{w} / \mathrm{w})$ was added. A hydrogen balloon was attached to the reaction flask. The heterogeneous reaction was stirred vigorously at room temperature $\left(25^{\circ} \mathrm{C}\right)$ for $5 \mathrm{~h}$. The reaction was filtered through a pad of celite and washed with EtOAc $(20 \mathrm{~mL} \times 3)$ and $\mathrm{CHCl}_{3}(20 \mathrm{~mL} \times 3)$. The filtrates were combined and concentrated in vacuo to yield bishydroquinone renieramycin $\mathrm{M}$ (8) as a colorless solid (quant). The product was employed in the next step without further purification. A solution of $8(10.0 \mathrm{mg}, 0.017 \mathrm{mmol}$, 1 equivalent $)$ in dry $\mathrm{CH}_{2} \mathrm{Cl}_{2}(2 \mathrm{~mL})$ was stirred at room temperature $\left(25^{\circ} \mathrm{C}\right)$ and EDCI.HCl $(3.3 \mathrm{mg}, 0.17$ mmol, 1 equivalent) and DMAP ( $2.1 \mathrm{mg}, 0.17 \mathrm{mmol}, 1$ equivalent) were added, followed by $N$-Boc-L-amino acid (10-25 mg, $0.085 \mathrm{mmol}, 5$ equivalent). The reaction mixture was stirred for $2 \mathrm{~h}$ in an argon atmosphere. 
The reaction mixture was quenched by the addition of water $(5 \mathrm{~mL})$. The organic layer was separated by a separating funnel and the aqueous layer was extracted with $\mathrm{CHCl}_{3}(10 \mathrm{~mL} \times 3)$. The organic layers were combined, washed with brine $(30 \mathrm{~mL})$, dried over anhydrous $\mathrm{Na}_{2} \mathrm{SO}_{4}$, filtered, and concentrated in vacuo. Purification by silica gel flash chromatography and eluting with n-hexane:EtOAc (3:1) gave hydroquinone 5-O-amino ester derivatives of $\mathbf{1}$.

5-O-(N-Boc-L-glycine) ester derivatives of renieramycin $\mathrm{M} \mathrm{(6a):} 4.8 \mathrm{mg}$ (51\%); yellow amorphous solid. $[\alpha]_{\mathrm{D}}^{25}+161.7\left(\mathrm{c}: 0.0007, \mathrm{CHCl}_{3}\right)$; CD $\Delta \varepsilon$ (c: $11 \mu \mathrm{M}$, methanol, $\left.20^{\circ} \mathrm{C}\right)+1.8(420), 0.0(384),-1.0(353)$, -0.4 (323), 0.0 (298), +1.9 (282), +3.1 (264), +2.0 (244), +1.5 (229), +2.6 (217); IR (KBr) 3393, 2961, 2926, 2855, $1775,1717,1653,1616,1462,1369,1261,1234,1153,1094,1059,1030,802 \mathrm{~cm}^{-1} ;{ }^{1} \mathrm{H}-\mathrm{NMR}\left(\mathrm{CDCl}_{3}, 400 \mathrm{MHz}\right)$ $\delta 5.93(1 \mathrm{H}, \mathrm{qq}, J=7.4,1.6 \mathrm{~Hz}, 26-\mathrm{H}), 5.70(1 \mathrm{H}, \mathrm{s}, 8-\mathrm{OH}), 5.00\left(1 \mathrm{H}, \mathrm{br} \mathrm{s}, 3^{\prime}-\mathrm{NH}\right), 4.44(1 \mathrm{H}, \mathrm{br} \mathrm{d}, J=11.0$ $\left.\mathrm{Hz}, 22-\mathrm{H}_{\mathrm{a}}\right), 4.24\left(2 \mathrm{H}, \mathrm{d}, J=4.4 \mathrm{~Hz}, 2^{\prime}-\mathrm{H}_{2}\right), 4.16(1 \mathrm{H}, \mathrm{br} \mathrm{d}, J=5.0 \mathrm{~Hz}, 1-\mathrm{H}), 4.04(1 \mathrm{H}, \mathrm{d}, J=2.8 \mathrm{~Hz}, 21-\mathrm{H})$, $3.98\left(1 \mathrm{H}, \mathrm{dd}, J=11.0,5.0 \mathrm{~Hz}, 22-\mathrm{H}_{\mathrm{b}}\right), 3.90\left(3 \mathrm{H}, \mathrm{s}, 17-\mathrm{OCH}_{3}\right), 3.88(1 \mathrm{H}, \mathrm{br} \mathrm{d}, J=2.2 \mathrm{~Hz}, 11-\mathrm{H}), 3.71(3 \mathrm{H}, \mathrm{s}$, $\left.7-\mathrm{OCH}_{3}\right), 3.29(1 \mathrm{H}, \mathrm{br} \mathrm{d}, J=8.0 \mathrm{~Hz}, 13-\mathrm{H}), 3.12(1 \mathrm{H}, \mathrm{dt}, J=12.2,2.2 \mathrm{~Hz}, 3-\mathrm{H}), 2.68(1 \mathrm{H}, \mathrm{dd}, J=21.4,8.0$ $\left.\mathrm{Hz}, 14-\mathrm{H}_{\alpha}\right), 2.40\left(1 \mathrm{H}, \mathrm{dd}, J=15.2,2.2 \mathrm{~Hz}, 4-\mathrm{H}_{\alpha}\right), 2.22\left(1 \mathrm{H}, \mathrm{d}, J=21.4 \mathrm{~Hz}, 14-\mathrm{H}_{\beta}\right), 2.20\left(3 \mathrm{H}, \mathrm{s}, \mathrm{NCH}_{3}\right)$, $2.02\left(3 \mathrm{H}, \mathrm{s}, 6-\mathrm{CH}_{3}\right), 1.86\left(3 \mathrm{H}, \mathrm{s}, 16-\mathrm{CH}_{3}\right), 1.80\left(3 \mathrm{H}, \mathrm{d}, J=7.4 \mathrm{~Hz}, 27-\mathrm{H}_{3}\right), 1.61\left(3 \mathrm{H}, \mathrm{br} \mathrm{s}, 28-\mathrm{H}_{3}\right), 1.55(1 \mathrm{H}$, overlapped, $\left.4-\mathrm{H}_{\beta}\right), 1.42\left(9 \mathrm{H}, \mathrm{s}, 3 \times 66^{\prime}-\mathrm{CH}_{3}\right) ;{ }^{13} \mathrm{C}-\mathrm{NMR}\left(\mathrm{CDCl}_{3}, 100 \mathrm{MHz}\right) \delta 186.1(\mathrm{C}-15), 182.8(\mathrm{C}-18), 180.9$ (C-1'), 167.1 (C-24), 155.4 (C-4'), 155.4 (C-17), 143.8 (C-7), 143.3 (C-8), 141.9 (C-20), 140.1 (C-26), 138.7 (C-5), 135.4 (C-19), 129.7 (C-16), 126.8 (C-25), 124.1 (C-10), 122.5 (C-6), 117.5 (21-CN), 117.2 (C-9), 80.2 (C-5'), 64.3 (C-22), $61.2\left(17-\mathrm{OCH}_{3}\right), 60.8\left(7-\mathrm{OCH}_{3}\right), 59.4(\mathrm{C}-21), 56.5(\mathrm{C}-1), 55.2(\mathrm{C}-3), 54.8$ (C-13), $54.8(\mathrm{C}-11), 42.2\left(\mathrm{C}-2^{\prime}\right)$, $41.5\left(\mathrm{NCH}_{3}\right), 28.3\left(3 \times 6^{\prime}-\mathrm{CH}_{3}\right), 27.9(\mathrm{C}-4), 21.1(\mathrm{C}-14), 20.6\left(28-\mathrm{CH}_{3}\right), 15.8\left(27-\mathrm{CH}_{3}\right), 10.0\left(16-\mathrm{CH}_{3}\right), 8.7$ $\left(6-\mathrm{CH}_{3}\right) \cdot \mathrm{FAB}^{+} \mathrm{HRMS} m / z 735.3250\left([\mathrm{M}+\mathrm{H}]^{+}\right.$, calculated for $\left.\mathrm{C}_{38} \mathrm{H}_{47} \mathrm{~N}_{4} \mathrm{O}_{11}, 735.3242\right)$.

5-O-(N-Boc-L-alanine) ester of renieramycin $\mathrm{M}(6 \mathbf{b}): 7.8 \mathrm{mg}(43 \%)$; yellow amorphous solid. $[\alpha]_{\mathrm{D}}^{25}$ +34.1 (c: 0.0039, $\left.\mathrm{CHCl}_{3}\right)$; CD $\Delta \varepsilon$ (c: $11 \mu \mathrm{M}$, methanol, $\left.20^{\circ} \mathrm{C}\right)+1.7(401),-0.4(381),-1.7(357),-0.5(331)$, +0.8 (307), +1.9 (294), +5.2 (282), +7.0 (267), +5.4 (250), +2.4 (236); IR (KBr) 3393, 2976, 2938, 1765, 1717, 1653, 1616, 1458, 1369, 1304, 1234, 1153, 1059, $1059 \mathrm{~cm}^{-1} ;{ }^{1} \mathrm{H}-\mathrm{NMR}\left(\mathrm{CDCl}_{3}, 400 \mathrm{MHz}\right) \delta 6.01(1 \mathrm{H}, \mathrm{qq}, J=7.4$, $1.6 \mathrm{~Hz}, 26-\mathrm{H}), 5.78(1 \mathrm{H}, \mathrm{s}, 8-\mathrm{OH}), 5.00\left(1 \mathrm{H}, \mathrm{br} \mathrm{s}, 3^{\prime}-\mathrm{NH}\right), 4.59\left(1 \mathrm{H}, \mathrm{m}, 2^{\prime}-\mathrm{H}\right), 4.48(1 \mathrm{H}, \mathrm{dd}, J=11.2,2.8 \mathrm{~Hz}$, $\left.22-\mathrm{H}_{\mathrm{a}}\right), 4.31(1 \mathrm{H}, \mathrm{dd}, J=5.4,2.8 \mathrm{~Hz}, 1-\mathrm{H}), 4.11(1 \mathrm{H}, \mathrm{d}, J=2.4 \mathrm{~Hz}, 21-\mathrm{H}), 4.02(1 \mathrm{H}, \mathrm{dd}, J=11.2,5.4 \mathrm{~Hz}$, $\left.22-\mathrm{H}_{\mathrm{b}}\right), 3.97\left(3 \mathrm{H}, \mathrm{s}, 17-\mathrm{OCH}_{3}\right), 3.94(1 \mathrm{H}, \mathrm{br} \mathrm{d}, J=2.6 \mathrm{~Hz}, 11-\mathrm{H}), 3.78\left(3 \mathrm{H}, \mathrm{s}, 7-\mathrm{OCH}_{3}\right), 3.35(1 \mathrm{H}, \mathrm{br} \mathrm{d}, J=7.4$ $\mathrm{Hz}, 13-\mathrm{H}), 3.20(1 \mathrm{H}, \mathrm{dt}, J=12.2,2.6 \mathrm{~Hz}, 3-\mathrm{H}), 2.75\left(1 \mathrm{H}, \mathrm{dd}, J=21.1,7.4 \mathrm{~Hz}, 14-\mathrm{H}_{\alpha}\right), 2.54(1 \mathrm{H}, \mathrm{br} \mathrm{dd}, J=15.2$, $\left.2.6 \mathrm{~Hz}, 4-\mathrm{H}_{\alpha}\right), 2.28\left(1 \mathrm{H}, \mathrm{d}, J=21.1 \mathrm{~Hz}, 14-\mathrm{H}_{\beta}\right), 2.27\left(3 \mathrm{H}, \mathrm{s}, \mathrm{NCH}_{3}\right), 2.11\left(3 \mathrm{H}, \mathrm{s}, 6-\mathrm{CH}_{3}\right), 1.92\left(3 \mathrm{H}, \mathrm{s}, 16-\mathrm{CH}_{3}\right)$, $1.88\left(3 \mathrm{H}, \mathrm{dq}, J=7.4,1.6 \mathrm{~Hz}, 27-\mathrm{H}_{3}\right), 1.72\left(3 \mathrm{H}, \mathrm{d}, J=7.2 \mathrm{~Hz}, 7^{\prime}-\mathrm{H}_{3}\right), 1.71\left(3 \mathrm{H}, \mathrm{br} \mathrm{s}, 28-\mathrm{H}_{3}\right), 1.64(1 \mathrm{H}, \mathrm{dd}$, $\left.J=15.2,12.2 \mathrm{~Hz}, 4-\mathrm{H}_{\beta}\right), 1.47\left(9 \mathrm{H}, \mathrm{s}, 3 \times 6^{\prime}-\mathrm{CH}_{3}\right) ;{ }^{13} \mathrm{C}-\mathrm{NMR}\left(\mathrm{CDCl}_{3}, 100 \mathrm{MHz}\right) \delta 186.1(\mathrm{C}-15), 182.8(\mathrm{C}-18)$, $171.6\left(\mathrm{C}-1^{\prime}\right), 167.1$ (C-24), 155.2 (C-17), $155.0\left(\mathrm{C}-4^{\prime}\right), 143.8$ (C-7), 143.3 (C-8), 142.0 (C-20), 140.0 (C-26), 138.7 (C-5), 135.4 (C-19), 129.0 (C-16), 126.8 (C-25), 124.1 (C-10), 122.7 (C-6), 117.5 (21-CN), 117.1 (C-9), 80.1 (C-5'), $64.8(\mathrm{C}-22), 61.2\left(17-\mathrm{OCH}_{3}\right), 60.8\left(7-\mathrm{OCH}_{3}\right), 59.7(\mathrm{C}-21), 56.4(\mathrm{C}-1), 55.4(\mathrm{C}-3), 54.8$ (C-13), 54.8 (C-11), 49.3 $\left(\mathrm{C}-2^{\prime}\right), 41.5\left(\mathrm{NCH}_{3}\right), 28.3\left(3 \times 6^{\prime}-\mathrm{CH}_{3}\right), 27.5(\mathrm{C}-4), 21.2(\mathrm{C}-14), 20.6\left(28-\mathrm{CH}_{3}\right), 18.7\left(7^{\prime}-\mathrm{CH}_{3}\right), 15.8\left(27-\mathrm{CH}_{3}\right)$, $10.1\left(16-\mathrm{CH}_{3}\right), 8.7\left(6-\mathrm{CH}_{3}\right)$. FAB ${ }^{+}$HRMS $m / z 749.3392\left([\mathrm{M}+\mathrm{H}]^{+}\right.$, calculated for $\left.\mathrm{C}_{39} \mathrm{H}_{49} \mathrm{~N}_{4} \mathrm{O}_{11}, 749.3398\right)$.

5-O-(N-Boc-L-phenylalanine) ester of renieramycin $\mathrm{M}(6 \mathrm{c})$ : $5.5 \mathrm{mg}$ (30\%); yellow amorphous solid. $[\alpha]_{\mathrm{D}}^{25}-28.1\left(\mathrm{c:} 0.0031, \mathrm{CHCl}_{3}\right) ; \mathrm{CD} \Delta \varepsilon\left(\mathrm{c}: 5 \mu \mathrm{M}\right.$, methanol, $\left.20^{\circ} \mathrm{C}\right)-6.1(358),-3.4(330),+0.2(299),+3.9$ (288), +15.6 (271), +11.7 (254), +3.5 (235), +18.6 (227), +30.7 (219), +24.9 (209); IR (KBr) 3393, 2934, 1763, $1717,1653,1616,1499,1456,1368,1303,1234,1161,1078,1059,770,700 \mathrm{~cm}^{-1} ;{ }^{1} \mathrm{H}-\mathrm{NMR}\left(\mathrm{CDCl}_{3}, 400 \mathrm{MHz}\right) \delta$ $7.38\left(3 \mathrm{H}, \mathrm{m}, 2 \times 10^{\prime}-\mathrm{H} \& 11^{\prime}-\mathrm{H}\right), 7.33\left(2 \mathrm{H}, \mathrm{dt}, J=8.8,2.8 \mathrm{~Hz}, 2 \times 9^{\prime}-\mathrm{H}\right), 6.01(1 \mathrm{H}, \mathrm{qq}, J=7.4,1.6 \mathrm{~Hz}, 26-\mathrm{H})$, $5.78(1 \mathrm{H}, \mathrm{s}, 8-\mathrm{OH}), 4.87\left(1 \mathrm{H}, \mathrm{br} \mathrm{d}, J=8.0 \mathrm{~Hz}, 3^{\prime}-\mathrm{NH}\right), 4.81\left(1 \mathrm{H}, \mathrm{br}, J=10.0,4.0 \mathrm{~Hz}, 2^{\prime}-\mathrm{H}\right), 4.48(1 \mathrm{H}, \mathrm{dd}, J=$ $\left.10.8,2.0 \mathrm{~Hz}, 22-\mathrm{H}_{\mathrm{a}}\right), 4.32(1 \mathrm{H}, \mathrm{t}, J=5.0 \mathrm{~Hz}, 1-\mathrm{H}), 4.10(1 \mathrm{H}, \mathrm{d}, J=2.0 \mathrm{~Hz}, 21-\mathrm{H}), 4.03(1 \mathrm{H}, \mathrm{dd}, J=10.8,5.0 \mathrm{~Hz}$, $\left.22-\mathrm{H}_{\mathrm{b}}\right), 3.94(1 \mathrm{H}, \mathrm{d}, J=2.4 \mathrm{~Hz}, 11-\mathrm{H}), 3.90\left(3 \mathrm{H}, \mathrm{s}, 17-\mathrm{OCH}_{3}\right), 3.78\left(3 \mathrm{H}, \mathrm{s}, 7-\mathrm{OCH}_{3}\right), 3.56(1 \mathrm{H}, \mathrm{dd}, J=13.8,4.4$ $\left.\mathrm{Hz}, 7^{\prime}-\mathrm{H}_{\mathrm{a}}\right), 3.35(1 \mathrm{H}, \mathrm{br} \mathrm{d}, J=7.6 \mathrm{~Hz}, 13-\mathrm{H}), 3.20(1 \mathrm{H}, \mathrm{dt}, J=12.0,2.4 \mathrm{~Hz}, 3-\mathrm{H}), 3.08(1 \mathrm{H}, \mathrm{dd}, J=13.8,10.2$ $\left.\mathrm{Hz}, 7^{\prime}-\mathrm{H}_{\mathrm{b}}\right), 2.74\left(1 \mathrm{H}, \mathrm{dd}, J=20.7,7.6 \mathrm{~Hz}, 14-\mathrm{H}_{\alpha}\right), 2.62\left(1 \mathrm{H}, \mathrm{br} \mathrm{d}, J=13.6 \mathrm{~Hz}, 4-\mathrm{H}_{\alpha}\right), 2.29(1 \mathrm{H}, \mathrm{d}, J=20.7 \mathrm{~Hz}$, 
$\left.14-\mathrm{H}_{\beta}\right), 2.26\left(3 \mathrm{H}, \mathrm{s}, \mathrm{NCH}_{3}\right), 2.09\left(3 \mathrm{H}, \mathrm{s}, 6-\mathrm{CH}_{3}\right), 1.92\left(3 \mathrm{H}, \mathrm{s}, 16-\mathrm{CH}_{3}\right), 1.88\left(3 \mathrm{H}, \mathrm{dq}, J=7.6,1.6 \mathrm{~Hz}, 27-\mathrm{H}_{3}\right)$, $1.71\left(3 \mathrm{H}, \mathrm{d}, J=1.6 \mathrm{~Hz}, 28-\mathrm{H}_{3}\right), 1.67\left(1 \mathrm{H}, \mathrm{dd}, J=13.6,12.0 \mathrm{~Hz}, 4-\mathrm{H}_{\beta}\right), 1.38\left(9 \mathrm{H}, \mathrm{s}, 3 \times 6^{\prime}-\mathrm{CH}_{3}\right) ;{ }^{13} \mathrm{C}-\mathrm{NMR}$ $\left(\mathrm{CDCl}_{3}, 100 \mathrm{MHz}\right) \delta 186.1$ (C-15), 182.8 (C-18), 170.1 (C-1'), 167.1 (C-24), $155.2\left(\mathrm{C}-4^{\prime}\right), 155.2$ (C-17), 143.8 (C-7), 143.3 (C-8), 142.0 (C-20), 140.0 (C-26), 138.8 (C-5), 136.3 (C-19), 135.4 (C-8'), 129.3 (2 × C-10'), 128.7 (C-16), $128.7\left(2 \times \mathrm{C}-9^{\prime}\right), 127.1\left(\mathrm{C}-11^{\prime}\right), 126.8(\mathrm{C}-25), 124.1$ (C-10), 123.1 (C-6), 117.6 (21-CN), 117.6 (C-9), 80.1 $\left(\mathrm{C}-5^{\prime}\right), 64.9(\mathrm{C}-22), 61.2\left(17-\mathrm{OCH}_{3}\right), 60.8\left(7-\mathrm{OCH}_{3}\right), 59.7\left(\mathrm{C}-2^{\prime}\right), 59.7$ (C-21), $56.4(\mathrm{C}-1), 55.5(\mathrm{C}-3), 54.9(\mathrm{C}-13)$, $54.9(\mathrm{C}-11), 41.5\left(\mathrm{NCH}_{3}\right), 38.3\left(\mathrm{C}-7^{\prime}\right), 28.2\left(3 \times 6^{\prime}-\mathrm{CH}_{3}\right), 27.1(\mathrm{C}-4), 21.2(\mathrm{C}-14), 20.6\left(28-\mathrm{CH}_{3}\right), 15.9\left(27-\mathrm{CH}_{3}\right)$, $10.1\left(16-\mathrm{CH}_{3}\right), 8.7\left(6-\mathrm{CH}_{3}\right)$. FAB ${ }^{+}$HRMS $m / z 825.3715\left([\mathrm{M}+\mathrm{H}]^{+}\right.$, calculated for $\left.\mathrm{C}_{45} \mathrm{H}_{53} \mathrm{~N}_{4} \mathrm{O}_{11}, 825.3712\right)$.

5-O-(N-Boc-L-valine derivative of renieramycin $\mathrm{M}(6 \mathrm{~d}): 4.0 \mathrm{mg}(30 \%)$; yellow amorphous solid. $[\alpha]_{\mathrm{D}}^{25}$ -11.5 (c: $\left.0.0026, \mathrm{CHCl}_{3}\right)$; $\mathrm{CD} \Delta \varepsilon$ (c: $10 \mu \mathrm{M}$, methanol, $\left.20^{\circ} \mathrm{C}\right)-1.8(400),-1.8$ (386), $-1.7(369),-1.3(348)$, -0.5 (325), -0.4 (298), -0.6 (288), -1.8 (273), -4.0 (251), -3.5 (240), -3.0 (229); IR (KBr) 3393, 2972, 2940, $1761,1717,1653,1616,1462,1369,1306,1234,1153 \mathrm{~cm}^{-1},{ }^{1} \mathrm{H}-\mathrm{NMR}\left(\mathrm{CDCl}_{3}, 400 \mathrm{MHz}\right) \delta 6.01(1 \mathrm{H}, \mathrm{qq}, J=7.6$, $1.6 \mathrm{~Hz}, 26-\mathrm{H}), 5.76(1 \mathrm{H}, \mathrm{s}, 8-\mathrm{OH}), 4.97\left(1 \mathrm{H}, \mathrm{d}, J=9.2 \mathrm{~Hz}, 3^{\prime}-\mathrm{NH}\right), 4.51\left(1 \mathrm{H}, \mathrm{dd}, J=9.6,4.8 \mathrm{~Hz}, 2^{\prime}-\mathrm{H}\right), 4.39$ $\left(1 \mathrm{H}, \mathrm{dd}, J=9.6,3.0 \mathrm{~Hz}, 22-\mathrm{H}_{\mathrm{a}}\right), 4.33(1 \mathrm{H}, \mathrm{dd}, J=5.6,3.0 \mathrm{~Hz}, 1-\mathrm{H}), 4.07(1 \mathrm{H}, \mathrm{d}, J=2.4 \mathrm{~Hz}, 21-\mathrm{H}), 3.99(1 \mathrm{H}$, overlapped, $\left.J=4.8 \mathrm{~Hz}, 22-\mathrm{H}_{\mathrm{b}}\right), 3.96\left(3 \mathrm{H}, \mathrm{s}, 17-\mathrm{OCH}_{3}\right), 3.95(1 \mathrm{H}$, overlapped, $11-\mathrm{H}), 3.77\left(3 \mathrm{H}, \mathrm{s}, 7-\mathrm{OCH}_{3}\right)$, $3.34(1 \mathrm{H}, \mathrm{br} \mathrm{d}, J=7.8 \mathrm{~Hz}, 13-\mathrm{H}), 3.20(1 \mathrm{H}, \mathrm{dt}, J=12.4,2.0 \mathrm{~Hz}, 3-\mathrm{H}), 2.74\left(1 \mathrm{H}, \mathrm{dd}, J=20.7,7.8 \mathrm{~Hz}, 14-\mathrm{H}_{\alpha}\right)$, $2.66\left(1 \mathrm{H}, \mathrm{d}, J=16.0 \mathrm{~Hz}, 4-\mathrm{H}_{\alpha}\right), 2.45\left(1 \mathrm{H}, \mathrm{m}, 7^{\prime}-\mathrm{H}\right), 2.31\left(1 \mathrm{H}\right.$, overlapped, 14- $\left.\mathrm{H}_{\beta}\right), 2.26\left(3 \mathrm{H}, \mathrm{s}, \mathrm{NCH}_{3}\right), 2.08$ $\left(3 \mathrm{H}, \mathrm{s}, 6-\mathrm{CH}_{3}\right), 1.92\left(3 \mathrm{H}, \mathrm{s}, 16-\mathrm{CH}_{3}\right), 1.85\left(3 \mathrm{H}, \mathrm{dq}, J=7.6,1.3 \mathrm{~Hz}, 27-\mathrm{H}_{3}\right), 1.71\left(3 \mathrm{H}, \mathrm{br} \mathrm{s}, 28-\mathrm{H}_{3}\right), 1.67(1 \mathrm{H}$, $\left.\mathrm{d}, J=16.0 \mathrm{~Hz}, 4-\mathrm{H}_{\beta}\right), 1.47\left(9 \mathrm{H}, \mathrm{s}, 3 \times 6^{\prime}-\mathrm{CH}_{3}\right), 1.19 \& 1.08\left(\right.$ each $\left.3 \mathrm{H}, \mathrm{d}, J=5.2 \mathrm{~Hz}, 2 \times 8^{\prime}-\mathrm{H} 3\right) ;{ }^{13} \mathrm{C}-\mathrm{NMR}$ $\left(\mathrm{CDCl}_{3}, 100 \mathrm{MHz}\right) \delta 186.2$ (C-15), 182.5 (C-18), $178.1\left(\mathrm{C}-1^{\prime}\right), 167.1$ (C-24), 155.8 (C-4'), 155.2 (C-17), 143.9 (C-7), 143.3 (C-8), 142.0 (C-20), 140.0 (C-26), 138.9 (C-5), 135.4 (C-19), 128.9 (C-16), 126.8 (C-25), 124.3 (C-10), 123.1 (C-6), $117.5(21-\mathrm{CN}), 117.2(\mathrm{C}-9), 80.1\left(\mathrm{C}-5^{\prime}\right), 65.4(\mathrm{C}-22), 61.2\left(17-\mathrm{OCH}_{3}\right), 60.9\left(7-\mathrm{OCH}_{3}\right), 60.1\left(\mathrm{C}-2^{\prime}\right)$, 58.8 (C-21), $56.3(\mathrm{C}-1), 55.9$ (C-3), $54.9(\mathrm{C}-13), 54.9(\mathrm{C}-11), 41.5\left(\mathrm{NCH}_{3}\right), 30.7\left(\mathrm{C}-7^{\prime}\right), 28.3\left(3 \times 6^{\prime}-\mathrm{CH}_{3}\right), 27.3$ $(\mathrm{C}-4), 21.4(\mathrm{C}-14), 20.6\left(28-\mathrm{CH}_{3}\right), 19.9\left(\mathrm{C}-8^{\prime}\right), 15.8\left(27-\mathrm{CH}_{3}\right), 10.3\left(16-\mathrm{CH}_{3}\right), 8.7\left(6-\mathrm{CH}_{3}\right) . \mathrm{FAB}^{+} \mathrm{HRMS} \mathrm{m} / \mathrm{z}$ $777.3705\left([\mathrm{M}+\mathrm{H}]^{+}\right.$, calculated for $\left.\mathrm{C}_{41} \mathrm{H}_{53} \mathrm{~N}_{4} \mathrm{O}_{11}, 777.3712\right)$.

5-O-acyl-N-Boc-L-glycine derivative of renieramycin $\mathrm{M}(6 \mathbf{e})$ : $4.1 \mathrm{mg}(27 \%)$; yellow amorphous solid. $[\alpha]_{\mathrm{D}}^{25}+92.0\left(\mathrm{c}: 0.0020, \mathrm{CHCl}_{3}\right) ; \mathrm{CD} \Delta \varepsilon\left(\mathrm{c}: 8 \mu \mathrm{M}\right.$, methanol, $\left.20^{\circ} \mathrm{C}\right) 0.0(299),+0.1(279),+0.3(263),+0.2(246)$, +0.3 (232), +0.5 (222); IR (KBr) 2978, 2938, 1717, 1655, 1616, 1506, 1456, 1369, 1308, 1261, 1236, 1161, 1109, 1094, 1061, 955, $802 \mathrm{~cm}^{-1} ;{ }^{1} \mathrm{H}-\mathrm{NMR}\left(\mathrm{CDCl}_{3}, 400 \mathrm{MHz}\right) \delta 5.99(1 \mathrm{H}, \mathrm{qq}, J=7.5,1.2 \mathrm{~Hz}, 26-\mathrm{H}), 4.63(2 \mathrm{H}, \mathrm{m}$, 22- $\left.\mathrm{H}_{2}\right), 4.59\left(1 \mathrm{H}\right.$, overlapped, $\left.2^{\prime}-\mathrm{H}\right), 4.28(1 \mathrm{H}, \mathrm{m}, 1-\mathrm{H}), 4.13(1 \mathrm{H}, \mathrm{d}, \mathrm{J}=2.4 \mathrm{~Hz}, 21-\mathrm{H}), 3.95\left(3 \mathrm{H}, \mathrm{s}, 17-\mathrm{OCH}_{3}\right)$, $3.90\left(1 \mathrm{H}\right.$, overlapped, 11-H), $3.71\left(3 \mathrm{H}, \mathrm{s}, 7-\mathrm{OCH}_{3}\right), 3.55(1 \mathrm{H}, \mathrm{br} \mathrm{d}, \mathrm{J}=7.6 \mathrm{~Hz}, 13-\mathrm{H}), 3.45\left(2 \mathrm{H}, \mathrm{m}, 9^{\prime}-\mathrm{H}_{2}\right), 3.19$ $(1 \mathrm{H}, \mathrm{br} \mathrm{d}, J=11.6,3-\mathrm{H}), 2.73\left(1 \mathrm{H}, \mathrm{dd}, J=21.0,7.6 \mathrm{~Hz}, 14-\mathrm{H}_{\alpha}\right), 2.52\left(1 \mathrm{H}\right.$, br d, $\left.J=14.4 \mathrm{~Hz}, 4-\mathrm{H}_{\alpha}\right), 2.32(1 \mathrm{H}$, $\left.\mathrm{d}, J=21.0 \mathrm{~Hz}, 14-\mathrm{H}_{\beta}\right), 2.25\left(3 \mathrm{H}, \mathrm{s}, \mathrm{NCH}_{3}\right), 2.17\left(2 \mathrm{H}\right.$, overlapped, $\left.8^{\prime}-\mathrm{H}\right), 2.09\left(3 \mathrm{H}, \mathrm{s}, 6-\mathrm{CH}_{3}\right), 1.93(2 \mathrm{H}, \mathrm{t}$, $\left.J=10 \mathrm{~Hz}, 7^{\prime}-\mathrm{H}_{2}\right), 1.91\left(3 \mathrm{H}, \mathrm{s}, 16-\mathrm{CH}_{3}\right), 1.87\left(3 \mathrm{H}, \mathrm{dq}, J=7.5,1.8 \mathrm{~Hz}, 27-\mathrm{H}_{3}\right), 1.69\left(3 \mathrm{H}, \mathrm{br} \mathrm{s}, 28-\mathrm{H}_{3}\right), 1.61(2 \mathrm{H}$, $\left.\mathrm{m}, 9^{\prime}-\mathrm{H}\right), 1.41\left(1 \mathrm{H}\right.$, overlapped, $\left.4-\mathrm{H}_{\beta}\right), 1.41\left(9 \mathrm{H}, \mathrm{s}, 6^{\prime}-\mathrm{CH}_{3}\right) ;{ }^{13} \mathrm{C}-\mathrm{NMR}\left(\mathrm{CDCl}_{3}, 100 \mathrm{MHz}\right) \delta 186.0(\mathrm{C}-15)$, 182.9 (C-18), 171.0 (C-1') 166.6 (C-24), 155.1 (C-4'), 154.4 (C-17), 144.1 (C-7), 148.8 (C-8), 142.2 (C-20), 139.7 (C-26), 138.6 (C-5), 135.2 (C-19), 129.8 (C-16), 126.8 (C-25), 124.7 (C-10), 124.5 (C-6), 117.3 (21-CN), 116.9 (C-9), $80.3\left(\mathrm{C}-5^{\prime}\right), 79.9(\mathrm{C}-22), 61.1\left(17-\mathrm{OCH}_{3}\right), 60.8\left(7-\mathrm{OCH}_{3}\right), 60.0\left(\mathrm{C}-2^{\prime}\right), 58.6(\mathrm{C}-21), 56.5\left(\mathrm{C}-9^{\prime}\right), 56.3(\mathrm{C}-1)$, 55.7 (C-3), 55.5 (C-13), 54.8 (C-11), $41.5\left(\mathrm{NCH}_{3}\right), 31.4\left(\mathrm{C}-7^{\prime}\right), 31.1\left(\mathrm{C}-8^{\prime}\right), 29.9(\mathrm{C}-4), 28.4\left(3 \times 6^{\prime}-\mathrm{CH}_{3}\right), 21.3$ (C-14), $20.6\left(28-\mathrm{CH}_{3}\right), 15.8\left(27-\mathrm{CH}_{3}\right), 10.5\left(16-\mathrm{CH}_{3}\right), 8.7\left(6-\mathrm{CH}_{3}\right)$. FAB ${ }^{+} \mathrm{HRMS} m / z 775.3550\left([\mathrm{M}+\mathrm{H}]^{+}\right.$, calculated for $\left.\mathrm{C}_{41} \mathrm{H}_{51} \mathrm{~N}_{4} \mathrm{O}_{11}, 775.3555\right)$.

\subsubsection{Cytotoxic Evaluations against Non-Small-Cell Lung Cancer Cell Lines}

The new series of 22-O-amino esters, $5 \mathbf{a}-\mathbf{5 e}$, and hydroquinone 5 -O-amino esters, $\mathbf{6 a - 6 e}$, were evaluated for their cytotoxicity against metastatic non-small cell lung carcinoma lines H292 and H460 according to the 3-(4,5-dimethylthiazol-2-yl)-2,5-diphenyltetrazolium bromide (MTT) assay [33]. Cisplatin 
and doxorubicin were used as the positive controls. A solution of $0.2 \%$ dimethylsulfoxide (DMSO) was used as the negative control. The samples were solubilized with $100 \%$ DMSO to $10 \mu \mathrm{M}$ and diluted to 0.5 $\mu \mathrm{M}$ with DMSO. Then, the resulting sample solutions were diluted with Roswell Park Memorial Institute (RPMI) medium to prepare the serial dilution. Non-small-cell lung cancer cells were seeded into a 96-well plate at a density of $2 \times 10^{3}$ cells/well and allow to adhere overnight. After that, cells were treated with various concentrations of each derivative that was dissolved in RPMI medium containing no more than $0.2 \%$ DMSO for $72 \mathrm{~h}$. Then, cells were incubated with $0.5 \mathrm{mg} / \mathrm{mL}$ MTT for $4 \mathrm{~h}$. The absorbance of the formazan products solubilized by DMSO was measured at $570 \mathrm{~nm}$. The percentage of cell viability was calculated with respect to non-treated control cells. The mean $\mathrm{IC}_{50}$ values \pm standard deviations (SD) were obtained from three independent experiments. Each experiment was performed in triplicate in at least five concentrations of the tested compounds on three different days. The $\mathrm{IC}_{50}$ value of each experiment was determined and averaged using GraphPad Prism software.

\section{Conclusions}

In summary, a new series of 22-O-amino ester and hydroquinone 5-O-amino ester derivatives of 1 was successfully prepared, staring from natural bis-1,2,3,4-tetrahydroisoquinolinequinone marine alkaloid (1) as the precursor. The chemical modification of 22-O-amino ester derivatives involved a reduction in the angelate ester (23-51\% yield) and esterification sequence (59-93\% yield), while the chemical modification of hydroquinone 5-O-amino ester derivatives involved hydrogenation and esterification (27-51\% yield of two steps). All synthesized compounds were tested for their cytotoxicity against the metastatic non-small-cell lung cancer $\mathrm{H} 292$ and $\mathrm{H} 460$ cell lines. The series of 22-O-amino ester derivatives were found to be more cytotoxic than that of hydroquinone 5- $\mathrm{O}$-amino ester derivatives. In addition, $22-\mathrm{O}$-amino ester derivatives showed a more promising cytotoxic activity against the H292 cell line than that of the H460 cell line. Among the compounds in these series, the most potent derivative is the 22-O-(N-Boc-L-glycine) ester of renieramycin $\mathrm{M}\left(5 \mathrm{a}, \mathrm{IC}_{50} 3.56 \mathrm{nM}\right)$, which was 7-fold more toxic than $\mathbf{1}\left(\mathrm{IC}_{50} 24.56 \mathrm{nM}\right)$ and 61 -fold more toxic than 7 ( $\left.\mathrm{IC}_{50} 217.43 \mathrm{nM}\right)$ against $\mathrm{H} 292$ cells. This new derivative will be further studied for its anti-lung cancer mechanism of action and its potentiality as a cytotoxic agent for the treatment of non-small-cell lung cancer.

Supplementary Materials: The following are available online at http://www.mdpi.com/1660-3397/18/8/418/s1, Figures S1-S20: ${ }^{1} \mathrm{H}$ and ${ }^{13} \mathrm{C}-\mathrm{NMR}$ spectra of $5 \mathbf{a}-\mathbf{5 e}$ and $\mathbf{6 a - 6 e}$.

Author Contributions: Conceptualization by S.C., P.C., K.S. and N.S. (Naoki Saito); methodology by S.C. and N.S. (Natchanun Sirimangkalakitti); investigation by S.C. and N.S. (Natchanun Sirimangkalakitti); writing-original draft preparation by S.C.; writing - review and editing by S.C., N.S. (Naoki Saito), P.C., K.S. and N.S. (Natchanun Sirimangkalakitti); supervision by S.C., P.C., K.S. and N.S. (Naoki Saito); project administration by S.C.; funding acquisition by S.C. and N.S. (Naoki Saito). All authors have read and agreed to the published version of the manuscript.

Funding: This research was funded by the Thai Research Fund, Thailand (RSA 6280009) to S.C. and was partially supported by a Grant-in-Aid (No. 18K06561) for Scientific Research from the Ministry of Education, Culture, Sports, Science, and Technology (MEXT) of Japan to N.S. (Naoki Saito).

Acknowledgments: The authors would like to thank the Natural Products and Nanoparticles Research Unit, NP2 (GRU 6306433004-1) and the Chulalongkorn Academic Advancement into Its Second Century (CUAASC) Project for their collaboration on the Development of Novel Drug Delivery Systems for Cancer Immunotherapy.

Conflicts of Interest: The authors declare no conflict of interest. 


\section{References}

1. Hu, Y.; Chen, J.; Hu, G.; Yu, J.; Zhu, X.; Lin, Y.; Chen, S.; Yuan, J. Statistical research on the bioactivity of new marine natural products discovered during the 28 years from 1985 to 2012. Mar. Drugs 2015, 13, $202-221$. [CrossRef] [PubMed]

2. Mayer, A.M.; Glaser, K.B.; Cuevas, C.; Jacobs, R.S.; Kem, W.; Little, R.D.; McIntosh, J.M.; Newman, D.J.; Potts, B.C.; Shuster, D.E. The odyssey of marine pharmaceuticals: A current pipeline perspective. Trends Pharmacol. Sci. 2010, 31, 255-265. [CrossRef] [PubMed]

3. Kim, S.-K. Handbook of Anticancer Drugs from Marine Origin; Springer: Berlin/Heidelberg, Germany, 2014.

4. Newman, D.; Cragg, G. Current status of marine-derived compounds as warheads in anti-tumor drug candidates. Mar. Drugs 2017, 15, 99. [CrossRef] [PubMed]

5. Khalifa, S.A.M.; Elias, N.; Farag, M.A.; Chen, L.; Saeed, A.; Hegazy, M.-E.F.; Moustafa, M.S.; Abd El-Wahed, A.; Al-Mousawi, S.M.; Musharraf, S.G.; et al. Marine natural products: A source of novel anticancer drugs. Mar. Drugs 2019, 17, 491. [CrossRef] [PubMed]

6. Jimenez, P.C.; Wilke, D.V.; Branco, P.C.; Bauermeister, A.; Rezende-Teixeira, P.; Gaudêncio, S.P.; Costa-Lotufo, L.V. Enriching cancer pharmacology with drugs of marine origin. Br. J. Pharmacol. 2020, 177, 3-27. [CrossRef]

7. Newman, D.J.; Cragg, G.M. Marine-sourced anti-cancer and cancer pain control agents in clinical and late preclinical development. Mar. Drugs 2014, 12, 255-278. [CrossRef]

8. Mayer, A.M.; Nguyen, M.; Kalwajtys, P.; Kerns, H.; Newman, D.J.; Glaser, K.B. The marine pharmacology and pharmaceuticals pipeline in 2016. FASEB J. 2017, 31, 818.1.

9. Scott, J.D.; Williams, R.M. Chemistry and biology of the tetrahydroisoquinoline antitumor antibiotics. Chem. Rev. 2002, 102, 1669-1730. [CrossRef]

10. Avendaño, C.; de la Cuesta, E. Recent synthetic approaches to 6,15-iminoisoquino[3,2-b]3-benzazocine compounds. Eur. J. Chem. 2010, 16, 9722-9734. [CrossRef]

11. Lane, J.W.; Estevez, A.; Mortara, K.; Callan, O.; Spencer, J.R.; Williams, R.M. Antitumor activity of tetrahydroisoquinoline analogues 3-epi-jorumycin and 3-epi-renieramycin G. Bioorg. Med. Chem. Lett. 2006, 16, 3180-3183. [CrossRef]

12. Le, V.H.; Inai, M.; Williams, R.M.; Kan, T. Ecteinascidins. A review of the chemistry, biology and clinical utility of potent tetrahydroisoquinoline antitumor antibiotics. Nat. Prod. Rep. 2015, 32, 328-347. [CrossRef]

13. Zewail-Foote, M.; Hurley, L.H. Ecteinascidin 743: A minor groove alkylator that bends DNA toward the major groove. J. Med. Chem. 1999, 42, 2493-2497.

14. Suwanborirux, K.; Amnuoypol, S.; Plubrukarn, A.; Pummangura, S.; Kubo, A.; Tanaka, C.; Saito, N. Chemistry of renieramycins. Part 3. Isolation and tructure of stabilized renieramycin type derivatives possessing antitumor activity from Thai sponge Xestospongia species, pretreated with potassium cyanide. J. Nat. Prod. 2003, 66, 1441-1446. [CrossRef]

15. Amnuoypol, S.; Suwanborirux, K.; Pummangura, S.; Kubo, A.; Tanaka, C.; Saito, N. Chemistry of renieramycins. Part 5. Structure elucidation of renieramycin-type derivatives $\mathrm{O}, \mathrm{Q}, \mathrm{R}$, and $\mathrm{S}$ from Thai marine sponge Xestospongia species pretreated with potassium cyanide. J. Nat. Prod. 2004, 67, 1023-1028. [CrossRef] [PubMed]

16. Charupant, K.; Daikuhara, N.; Saito, E.; Amnuoypol, S.; Suwanborirux, K.; Owa, T.; Saito, N. Chemistry of renieramycins. Part 8: Synthesis and cytotoxicity evaluation of renieramycin M-jorunnamycin A analogues. Bioorg. Med. Chem. 2009, 17, 4548-4558. [CrossRef] [PubMed]

17. Sirimangkalakitti, N.; Chamni, S.; Charupant, K.; Chanvorachote, P.; Mori, N.; Saito, N.; Suwanborirux, K. Chemistry of renieramycins. Part 15 . Synthesis of 22-O-ester derivatives of jorunnamycin A and their cytotoxicity against non-small-cell lung cancer Cells. J. Nat. Prod. 2016, 79, 2089-2093. [CrossRef] [PubMed]

18. Chamni, S.; Sirimangkalakitti, N.; Chanvorachote, P.; Saito, N.; Suwanborirux, K. Chemistry of renieramycins. Part 17. A new generation of renieramycins: Hydroquinone 5-O-monoester analogues of renieramycin $\mathrm{M}$ as potential cytotoxic agents against non-small-cell lung cancer cells. J. Nat. Prod. 2017, 80, 1541-1547. [CrossRef] [PubMed] 
19. Jemal, A.; Bray, F.; Center, M.M.; Ferlay, J.; Ward, E.; Forman, D. Global cancer statistics. CA Cancer J. Clin. 2011, 61, 69-90. [CrossRef]

20. WHO. The Top 10 Causes of Death Fact Sheet N³10; WHO: Geneva, Switzerland, 2014.

21. Halim, H.; Chunhacha, P.; Suwanborirux, K.; Chanvorachote, P. Anticancer and antimetastatic activities of renieramycin $\mathrm{M}$, a marine tetrahydroisoquinoline alkaloid, in human non-small cell lung cancer cells. Anticancer Res. 2011, 31, 193-201.

22. Cheun-Arom, T.; Chanvorachote, P.; Sirimangkalakitti, N.; Chuanasa, T.; Saito, N.; Abe, I.; Suwanborirux, K. Replacement of a quinone by a 5-O-acetylhydroquinone abolishes the accidental necrosis inducing effect while preserving the apoptosis-inducing effect of renieramycin M on lung cancer cells. J. Nat. Prod. 2013, 76, 1468-1474. [CrossRef]

23. Sirimangkalakitti, N.; Chamni, S.; Suwanborirux, K.; Chanvorachote, P. Renieramycin M sensitizes anoikis-resistant H460 lung cancer cells to anoikis. Anticancer Res. 2016, 36, 1665-1671. [PubMed]

24. Pinkhien, T.; Maiuthed, A.; Chamni, S.; Suwanborirux, K.; Saito, N.; Chanvorachote, P. Bishydroquinone renieramycin $\mathrm{M}$ induces apoptosis of human lung cancer cells through a mitochondria-dependent pathway. Anticancer Res. 2016, 36, 6327-6333. [CrossRef] [PubMed]

25. Sirimangkalakitti, N.; Chamni, S.; Suwanborirux, K.; Chanvorachote, P. Renieramycin M attenuates cancer stem cell-like phenotypes in H460 lung cancer cells. Anticancer Res. 2017, 37, 615-621. [CrossRef] [PubMed]

26. Maiuthed, A.; Pinkhien, T.; Chamni, S.; Suwanborirux, K.; Saito, N.; Petpiroon, N.; Chanvorachote, P. Apoptosis-inducing effect of hydroquinone 5-O-cinnamoyl ester analog of renieramycin $\mathrm{M}$ on non-small cell lung cancer cells. Anticancer Res. 2017, 37, 6259-6267.

27. Saito, N.; Tanaka, C.; Koizumi, Y.; Suwanborirux, K.; Amnuoypol, S.; Pummangura, S.; Kubo, A. Chemistry of renieramycins. Part 6: Transformation of renieramycin $\mathrm{M}$ into jorumycin and renieramycin J including oxidative degradation products, mimosamycin, renierone, and renierol acetate. Tetrahedron 2004, 60, 3873-3881. [CrossRef]

28. Neises, B.; Steglich, W. Simple Method for the Esterification of Carboxylic Acids. Angew. Chem. Int. Ed. Engl. 1978, 17, 522-524. [CrossRef]

29. Ghosh, A.K.; Brindisi, M.J. Organic carbamates in drug design and medicinal chemistry. J. Med. Chem. 2015, 58, 2895-2940.

30. Jornada, D.H.; dos Santos Fernandes, G.F.; Chiba, D.E.; De Melo, T.R.F.; Dos Santos, J.L.; Chung, M.C. The prodrug approach: A successful tool for improving drug solubility. Molecules 2016, 21, 42. [CrossRef]

31. Polakis, P. Antibody Drug Conjugates for Cancer Therapy. Pharmacol. Rev. 2016, 68, 3-19. [CrossRef]

32. Taylor, J. Toxicological Profile for Cyanide; U.S. Dept. of Health and Human Services, Public Health Service, Agency for Toxic Substances and Disease Registry: Atlanta, GA, USA, 2006.

33. Riss, T.L.; Moravec, R.A.; Niles, A.L.; Benink, H.A.; Worzella, T.J.; Minor, L. Assay Guidance Manual; Eli Lilly \& Company and the National Center for Advancing Translational Sciences: Bethesda, MD, USA, 2004.

(C) 2020 by the authors. Licensee MDPI, Basel, Switzerland. This article is an open access article distributed under the terms and conditions of the Creative Commons Attribution (CC BY) license (http://creativecommons.org/licenses/by/4.0/). 